\title{
COXETER COMBINATORICS FOR SUM FORMULAS IN THE REPRESENTATION THEORY OF ALGEBRAIC GROUPS
}

\author{
JONATHAN GRUBER
}

\begin{abstract}
Let $G$ be a simple algebraic group over an algebraically closed field $\mathbb{F}$ of characteristic $p \geq h$, the Coxeter number of $G$. We observe an easy 'recursion formula' for computing the Jantzen sum formula of a Weyl module with $p$-regular highest weight. We also discuss a 'duality formula' that relates the Jantzen sum formula to Andersen's sum formula for tilting filtrations and we give two different representation theoretic explanations of the recursion formula. As a corollary, we also obtain an upper bound on the length of the Jantzen filtration of a Weyl module with $p$-regular highest weight in terms of the length of the Jantzen filtration of a Weyl module with highest weight in an adjacent alcove.
\end{abstract}

\section{INTRODUCTION}

One of the most important problems in the representation theory of reductive algebraic groups in positive characteristic $p$ is to find the composition multiplicities of Weyl modules. When the characteristic is very large then these multiplicities can be computed as the values at 1 of certain Kazhdan-Lusztig polynomials 11 but the $p$-canonical basis that provides a replacement for the Kazhdan-Lusztig basis in arbitrary characteristid 2 quickly becomes prohibitively hard to compute in higher rank. For a specific weight $\lambda$, another tool for computing partial information about composition multiplicities in the Weyl module $\Delta(\lambda)$ is the Jantzen sum formula. By lifting to the $p$-adic integers a non-zero homomorphism from $\Delta(\lambda)$ to the induced module $\nabla(\lambda)$, one can define the Jantzen filtration, an exhaustive descending filtration

$$
\Delta(\lambda) \supseteq \Delta(\lambda)^{1} \supseteq \Delta(\lambda)^{2} \supseteq \cdots
$$

such that $\Delta(\lambda) / \Delta(\lambda)^{1}=L(\lambda)$ is the unique simple quotient of $\Delta(\lambda)$ and the sum of the characters of the submodules $\Delta(\lambda)^{i}$ for $i>0$ can be computed explicitly using the Jantzen sum formula. But again, the computation of this formula becomes quite tedious in large rank because it requires the determination of the dominant conjugates under the (finite) Weyl group $W_{\text {fin }}$ of $G$ of many non-dominant weights (with respect to the dot action).

Received by the editors July 7, 2021, and, in revised form, August 20, 2021.

2020 Mathematics Subject Classification. Primary 20 G05.

This work was supported by the Swiss National Science Foundation under grant number FNS 200020_175571.

${ }^{1}$ This was proved by a reduction from algebraic groups to quantum groups AJS94 and from quantum groups to affine Lie algebras KL93 KL94]. The case of affine Lie algebras was treated in KT95. In the non-simply-laced case, additional results from Lus94] and KT96] were required.

${ }^{2}$ To be more precise, the $p$-canonical basis determines the multiplicities in good filtrations of tilting modules RW20]. These in turn determine the characters of simple modules (and hence the composition multiplicities of Weyl modules) by the results of [Sob20]. 
In this paper, we give a 'recursion formula' that can be used to compute the Jantzen sum formula for a Weyl module with $p$-regular highest weight from the Jantzen sum formula for a Weyl module with highest weight in an adjacent alcove. It is convenient to identify the character lattice of a fixed $p$-regular linkage class with the anti-spherical module $M_{\text {asph }}$ over the integral group ring of the affine Weyl group $W_{\text {aff }}$ of $G$. The characters of the Weyl modules in the linkage class form a basis of its character lattice which is canonically indexed by the set $W_{\text {aff }}^{+}$of elements $x \in W_{\text {aff }}$ whose length is minimal in the coset $W_{\text {fin }} x$. These characters correspond to the standard basis $\left\{N_{x} \mid x \in W_{\text {aff }}^{+}\right\}$of $M_{\text {asph }}$ in the aforementioned identification. Similarly, the Jantzen sum formula for a Weyl module indexed by $x \in W_{\text {aff }}^{+}$corresponds to an element $\mathrm{JSF}_{x} \in M_{\text {asph }}$. With this notation in place, we can now state our recursion formula; see Section 2 for the definition of the affine reflection $s_{\beta, m} \in W_{\text {aff }}$.

Recursion formula. Let $x \in W_{\text {aff }}^{+}$and let $s \in W_{\text {aff }}$ be a simple reflection such that $x<x s$ in the Bruhat order and $x s \in W_{\text {aff }}^{+}$. Write $x s x^{-1}=s_{\beta, m}$ for some $m>0$ and a positive root $\beta$. Then

$$
\mathrm{JSF}_{x s}=\nu_{p}(m \cdot p) \cdot N_{x}+\mathrm{JSF}_{x} \cdot s .
$$

In And97, H. H. Andersen defined a filtration on the space of homomorphisms from a Weyl module to a tilting module. Its construction is similar to that of the Jantzen filtration and the sum of the dimensions of the subspaces appearing in the filtration is given by the Andersen sum formula. In Section 3 we explain how the Andersen sum formula for a fixed Weyl module indexed by $x \in W_{\text {aff }}^{+}$can be viewed as an element $\mathrm{ASF}_{x}$ of the dual $M_{\text {asph }}^{*}$ of the anti-spherical module. Writing

$$
\langle-,-\rangle: M_{\text {asph }}^{*} \times M_{\text {asph }} \longrightarrow \mathbb{Z}
$$

for the evaluation pairing and $\left\{N_{x}^{*} \mid x \in W_{\text {aff }}^{+}\right\}$for the 'dual basis' of the standard basis of $M_{\mathrm{asph}}$, we can relate the two sum formulas by a simple 'duality formula'.

Duality formula. For $x, y \in W_{\text {aff }}^{+}$, we have

$$
\left\langle\mathrm{ASF}_{x}, N_{y}\right\rangle=\left\langle N_{x}^{*}, \mathrm{JSF}_{y}\right\rangle .
$$

The formula is new in this formulation, but it has already appeared implicitly in work of H. H. Andersen and U. Kulkarni AK08, see Remark 4.3.

Both the recursion formula and the duality formula are purely combinatorial observations at first, so one might wonder if it is possible to find a representation theoretic explanation for them as well (or to categorify them, as one might say). We give two versions of such representation theoretic explanations in Section 5. The first one (Theorem 5.10) relates the cokernels of the natural maps from Weyl modules to induced modules with highest weights in adjacent alcoves (over the $p$-adic integers); these cokernels in turn determine the respective Jantzen sum formulas. The second one is via a 'torsion Euler characteristic' that had already been used by H. H. Andersen and U. Kulkarni to study the two sum formulas (see Lemma 5.14 and Remark 5.15). As a corollary of Theorem 5.10, we also obtain an upper bound on the length of the Jantzen filtration of a Weyl module with $p$-regular highest weight in terms of the length of the Jantzen filtration of a Weyl module with highest weight in an adjacent alcove. 
Corollary. Let $\lambda$ be a dominant weight in the fundamental alcove and let $x \in W_{\mathrm{aff}}^{+}$. Further let $s \in W_{\text {aff }}$ be a simple reflection such that $x<x s$ and $x s \in W_{\mathrm{aff}}^{+}$and write $x s x^{-1}=s_{\beta, m}$ for some $m>0$ and a positive root $\beta$. Then

$$
\max \left\{i \geq 0 \mid \Delta(x s \cdot \lambda)^{i} \neq 0\right\} \leq 2 \cdot \max \left\{i \geq 0 \mid \Delta(x \cdot \lambda)^{i} \neq 0\right\}+\nu_{p}(m \cdot p) .
$$

We give an example of how the recursion formula can be applied in Section 6 .

\section{Notation}

Let $G$ be a simply connected simple algebraic group over an algebraically closed field $\mathbb{F}$ of positive characteristic $p$. Let $X$ be the weight lattice of $G$ (with respect to some fixed maximal torus) and denote by $\Phi \subseteq X$ the root system of $G$ with positive system $\Phi^{+}$corresponding to a base $\Delta$ of $\Phi$. We write $X^{+}$for the set of dominant weights, $\rho$ for the half sum of positive roots and $\alpha_{\mathrm{h}}$ for the highest short root in $\Phi$. Let $W_{\text {fin }}$ be the (finite) Weyl group of $G$ and fix a $W_{\text {fin-invariant inner }}$ product $\langle-,-\rangle$ on $X_{\mathbb{R}}=X \otimes_{\mathbb{Z}} \mathbb{R}$. For $\alpha \in \Phi^{+}$, we write $\alpha^{\vee}=2 \alpha /\langle\alpha, \alpha\rangle$ for the coroot of $\alpha$ and $s_{\alpha} \in W_{\text {fin }}$ for the reflection with $s_{\alpha}(x)=x-\left\langle x, \alpha^{\vee}\right\rangle \cdot \alpha$ for $x \in X_{\mathbb{R}}$. We consider the dot action of $W_{\text {fin }}$ on $X_{\mathbb{R}}$, given by

$$
w \cdot x:=w(x+\rho)-\rho
$$

for $w \in W_{\text {fin }}$ and $x \in X_{\mathbb{R}}$.

We write $\operatorname{Rep}(G)$ for the category of finite-dimensional $G$-modules and for any finite-dimensional $G$-module $M$, we write $\operatorname{ch} M \in \mathbb{Z}[X]^{W_{\text {fin }}}$ for the character of $M$ and $[M]$ for the class of $M$ in the Grothendieck group $[\operatorname{Rep}(G)]$ of $\operatorname{Rep}(G)$. For $\lambda \in X^{+}$, we denote by $L(\lambda), \Delta(\lambda), \nabla(\lambda)$ and $T(\lambda)$ the simple module, the Weyl module, the induced module and the indecomposable tilting module of highest weight $\lambda$, respectively, and we write $\chi_{\lambda}=\operatorname{ch} \Delta(\lambda)=\operatorname{ch} \nabla(\lambda)$. See Sections II.2.14, II.2.13 and II.E.4 in Jan03 for the definitions of these modules and note that $\Delta(\lambda)$ and $\nabla(\lambda)$ are denoted by $V(\lambda)$ and $H^{0}(\lambda)$ there, respectively. The characters $\chi_{\lambda}$, for $\lambda \in X^{+}$, form a basis of the $\mathbb{Z}$-module $\mathbb{Z}[X]^{W_{\text {fin }}}$ and for a $G$-module $M$ with $\operatorname{ch} M=\sum_{\lambda} a_{\lambda} \cdot \chi_{\lambda}$, we define $\left[\operatorname{ch} M: \chi_{\lambda}\right]:=a_{\lambda}$. For arbitrary $\lambda \in X$, there exists a unique $\lambda^{\prime} \in W_{\text {fin }} \cdot \lambda$ with $\lambda^{\prime}+\rho \in X^{+}$and if $\lambda^{\prime} \in X^{+}$then there is a unique $w \in W_{\text {fin }}$ with $\lambda=w \cdot \lambda^{\prime}$. For such $\lambda^{\prime}$ and $w$, we define

$$
\chi_{\lambda}= \begin{cases}\operatorname{det}(w) \cdot \chi_{\lambda^{\prime}} & \text { if } \lambda^{\prime} \in X^{+}, \\ 0 & \text { otherwise }\end{cases}
$$

and

$$
\left[\operatorname{ch} M: \chi_{\lambda}\right]= \begin{cases}\operatorname{det}(w) \cdot\left[\operatorname{ch} M: \chi_{\lambda^{\prime}}\right] & \text { if } \lambda^{\prime} \in X^{+} \\ 0 & \text { otherwise. }\end{cases}
$$

For a $G$-module $M$ and an indecomposable $G$-module $N$, we write $[M: N]_{\oplus}$ for the multiplicity of $N$ in a Krull-Schmidt decomposition of $M$. Let $\mathbb{Z}_{p}$ be the ring of $p$-adic integers and denote by $G_{\mathbb{Z}_{p}}$ the algebraic group scheme over $\mathbb{Z}_{p}$ corresponding to $G$. For $\lambda \in X^{+}$, we can define a Weyl module $\Delta_{\mathbb{Z}_{p}}(\lambda)$ and an induced module $\nabla_{\mathbb{Z}_{p}}(\lambda)$ over $G_{\mathbb{Z}_{p}}$ such that both $\Delta_{\mathbb{Z}_{p}}(\lambda)$ and $\nabla_{\mathbb{Z}_{p}}(\lambda)$ are free over $\mathbb{Z}_{p}$ and $\Delta_{\mathbb{Z}_{p}}(\lambda) \otimes \mathbb{F} \cong \Delta(\lambda)$ and $\nabla_{\mathbb{Z}_{p}}(\lambda) \otimes \mathbb{F} \cong \nabla(\lambda)$ as $G$-modules. By Section II.B.4 in [Jan03], we have

$$
\operatorname{Ext}_{G_{\mathbb{Z}_{p}}}^{i}\left(\Delta_{\mathbb{Z}_{p}}(\lambda), \nabla_{\mathbb{Z}_{p}}(\mu)\right) \cong \begin{cases}\mathbb{Z}_{p} & \text { if } \lambda=\mu \text { and } i=0 \\ 0 & \text { otherwise }\end{cases}
$$


for all $\lambda, \mu \in X^{+}$and $i \geq 0$. For each $\lambda \in X^{+}$, we fix a generator $c_{\lambda}$ of $\operatorname{Hom}_{G_{\mathbb{Z}_{p}}}\left(\Delta_{\mathbb{Z}_{p}}(\lambda), \nabla_{\mathbb{Z}_{p}}(\mu)\right)$.

\section{Filtrations AND SUm FORMUlas}

For any homomorphism of $\mathbb{Z}_{p}$-modules $\varphi: M \rightarrow N$, we can define a descending filtration

$$
M=F^{0}(\varphi) \supseteq F^{1}(\varphi) \supseteq F^{2}(\varphi) \supseteq \cdots
$$

with $F^{i}(\varphi):=\varphi^{-1}\left(p^{i} \cdot N\right)$ for $i \geq 0$. For $\lambda \in X^{+}$and $i>0$, we write $\Delta(\lambda)^{i}$ for the submodule that is spanned by the image of $F^{i}\left(c_{\lambda}\right)$ in $\Delta(\lambda) \cong \Delta_{\mathbb{Z}_{p}}(\lambda) \otimes \mathbb{F}$ and call the descending filtration

$$
\Delta(\lambda)=\Delta(\lambda)^{0} \supseteq \Delta(\lambda)^{1} \supseteq \Delta(\lambda)^{2} \supseteq \cdots
$$

the Jantzen filtration. As $c_{\lambda}$ is unique up to a unit in $\mathbb{Z}_{p}$, this is independent of the choice of $c_{\lambda}$. Partial information about the layers of this filtration can be obtained from the following formula (see Section II.8.19 in [Jan03]):

Theorem 1.1 (Jantzen sum formula). For $\lambda \in X^{+}$, we have

$$
\mathrm{JSF}_{\lambda}:=\sum_{i>0} \operatorname{ch} \Delta(\lambda)^{i}=-\sum_{\alpha \in \Phi^{+}} \sum_{0<m<\left\langle\lambda+\rho, \alpha^{\vee}\right\rangle} \nu_{p}(m) \cdot \chi_{\lambda-m \alpha}
$$

and

$$
\Delta(\lambda) / \Delta(\lambda)^{1} \cong L(\lambda) .
$$

Now let $T$ be a tilting $G_{\mathbb{Z}_{p}}$-module. For $\lambda \in X^{+}$, we define

$$
F_{\lambda}(T):=\operatorname{Hom}_{G_{\mathbb{Z}_{p}}}\left(\Delta_{\mathbb{Z}_{p}}(\lambda), T\right) \quad \text { and } \quad E_{\lambda}(T):=\operatorname{Hom}_{G_{\mathbb{Z}_{p}}}\left(T, \nabla_{\mathbb{Z}_{p}}(\lambda)\right) .
$$

Both $F_{\lambda}(T)$ and $E_{\lambda}(T)$ are free over $\mathbb{Z}_{p}$, see Section II.E.23 in [Jan03. There is a canonical homomorphism of $\mathbb{Z}_{p}$-modules $d_{\lambda}: F_{\lambda}(T) \rightarrow E_{\lambda}(T)^{*}$ with

$$
e \circ f=d_{\lambda}(f)(e) \cdot c_{\lambda}
$$

for $f \in F_{\lambda}(T)$ and $e \in E_{\lambda}(T)$ and we define $F_{\lambda}^{i}(T):=F^{i}\left(d_{\lambda}\right)$. Now if $T$ is a tilting $G$-module then there is a tilting $G_{\mathbb{Z}_{p}}$-module $\hat{T}$ (unique up to isomorphism) with $T \cong \hat{T} \otimes \mathbb{F}$ and we write $\bar{F}_{\lambda}^{i}(T)$ for the subspace that is spanned by the image of $F_{\lambda}^{i}(\hat{T})$ in $\operatorname{Hom}_{G}(\Delta(\lambda), T) \cong F_{\lambda}(\hat{T}) \otimes \mathbb{F}$. The descending filtration

$$
\operatorname{Hom}_{G}(\Delta(\lambda), T)=\bar{F}_{\lambda}^{0}(T) \supseteq \bar{F}_{\lambda}^{1}(T) \supseteq \bar{F}_{\lambda}^{1}(T) \supseteq \cdots
$$

is called the Andersen filtration. As for the Jantzen filtration, there is a sum formula that gives partial information about the layers of the filtration; see Theorem 5.2 in AK08.

Theorem 1.2 (Andersen sum formula). For $\lambda \in X^{+}$and a tilting $G$-module $T$, we have

$$
\operatorname{ASF}(\lambda, T):=\sum_{i>0} \operatorname{dim} \bar{F}_{\lambda}^{i}(T)=-\sum_{\alpha \in \Phi^{+}} \sum_{m \notin I(\lambda, \alpha)} \nu_{p}(m) \cdot\left[\operatorname{ch} T: \chi_{\lambda-m \alpha}\right],
$$

where $I(\lambda, \alpha)=\left\{m \in \mathbb{Z} \mid 0 \leq m \leq\left\langle\lambda+\rho, \alpha^{\vee}\right\rangle\right\}$, and

$$
\operatorname{dim}\left(\bar{F}_{\lambda}^{0}(T) / \bar{F}_{\lambda}^{1}(T)\right)=[T: T(\lambda)]_{\oplus} .
$$

Note that the inner sum on the right-hand side of the Andersen sum formula is infinite, but the multiplicity $\left[\operatorname{ch} T: \chi_{\lambda-m \alpha}\right]$ is zero for all but finitely many values of $m$. 


\section{Reflections in the AfFine Weyl group}

The representation theory of $G$ is governed to a large extent by the affine Weyl group $W_{\text {aff }}$ of $G$ (to be defined below) and the alcove geometry associated with its $p$-dilated dot action. In order to formulate our main results, we need to introduce some more notation and establish certain properties of reflections in $W_{\text {aff }}$. Most of the material in this section is presumably well-known to experts.

The affine Weyl group of $G$ is the semidirect product $W_{\text {aff }}=\mathbb{Z} \Phi \rtimes W_{\text {fin }}$ and we write $\gamma \mapsto t_{\gamma}$ for the canonical embedding of $\mathbb{Z} \Phi$ into $W_{\text {aff. }}$. For $\alpha \in \Phi^{+}$ and $m \in \mathbb{Z}$, we define an affine reflection $s_{\alpha, m} \in W_{\text {aff }}$ by $s_{\alpha, m}:=t_{m \alpha} s_{\alpha}$. With $S=\left\{s_{\alpha} \mid \alpha \in \Delta\right\} \cup\left\{s_{\alpha_{\mathrm{h}}, 1}\right\}$, the pair $\left(W_{\text {aff }}, S\right)$ is a Coxeter system whose length function we denote by $\ell: W_{\text {aff }} \rightarrow \mathbb{Z}_{\geq 0}$; see Section II.6.3 in [Jan03] and the references therein. We write $W_{\text {aff }}^{+}$for the set of elements $x \in W_{\text {aff }}$ whose length is minimal in the coset $W_{\text {fin }} x$.

We consider the $p$-dilated dot action of $W_{\text {aff }}$ on $X_{\mathbb{R}}$, given by

$$
t_{\gamma} w \cdot x:=w(x+\rho)+p \cdot \gamma-\rho
$$

for $\gamma \in \mathbb{Z} \Phi, w \in W_{\text {fin }}$ and $x \in X_{\mathbb{R}}$. The fixed points of an affine reflection $s=s_{\alpha, m}$ (with respect to the $p$-dilated dot action) in $X_{\mathbb{R}}$ form an affine hyperplane

$$
H_{s}=H_{\alpha, m}^{p}=\left\{x \in X_{\mathbb{R}} \mid\left\langle x+\rho, \alpha^{\vee}\right\rangle=m p\right\}
$$

and the connected components of $X_{\mathbb{R}} \backslash \bigcup_{\alpha, m} H_{\alpha, m}^{p}$ are called the alcoves in $X_{\mathbb{R}}$. We say that a hyperplane $H$ separates two alcoves $C$ and $C^{\prime}$ if $C$ and $C^{\prime}$ are contained in different connected components of $X_{\mathbb{R}} \backslash H$ and we call two alcoves adjacent if they are separated by a unique hyperplane. The alcove

$$
C_{\text {fund }}=\left\{x \in X_{\mathbb{R}} \mid 0<\left\langle x+\rho, \alpha^{\vee}\right\rangle<p \text { for all } \alpha \in \Phi^{+}\right\}
$$

is called the fundamental alcove. The closure $\bar{C}_{\text {fund }}$ is a fundamental domain for the action of $W_{\text {aff }}$ on $X_{\mathbb{R}}$ and we call a dominant weight $\lambda \in X^{+}$p-regular if $\lambda \in x \cdot C_{\text {fund }}$ for some $x \in W_{\text {aff }}$. By the linkage principle (see Section II.6 in [Jan03), the category $\operatorname{Rep}(G)$ of finite dimensional $G$-modules decomposes as

$$
\operatorname{Rep}(G)=\bigoplus_{\lambda \in \bar{C}_{\text {fund }} \cap X} \operatorname{Rep}_{\lambda}(G)
$$

where $\operatorname{Rep}_{\lambda}(G)$ denotes the linkage class of $\lambda \in \bar{C}_{\text {fund }} \cap X$, the full subcategory of $\operatorname{Rep}(G)$ consisting of the $G$-modules $M$ such that all composition factors of $M$ are of the form $L(w \cdot \lambda)$ for some $w \in W_{\text {aff }}$.

Now let us write $R\left(W_{\text {aff }}\right)=\left\{s_{\alpha, m} \mid \alpha \in \Phi^{+}, m \in \mathbb{Z}\right\}$ for the set of reflections in $W_{\text {aff }}$ and $R\left(W_{\text {fin }}\right)$ for the set of reflections in $W_{\text {fin }}$. For $y \in W_{\text {aff }}$, we will be interested in sets of the form

$$
R_{L}(y):=\left\{s \in R\left(W_{\mathrm{aff}}\right) \mid s y<y\right\},
$$

where $<$ denotes the Bruhat order. By Corollaries 1.4.4 and 1.4.5 in BB05, we have $\left|R_{L}(y)\right|=\ell(y)$ and if $y=s_{1} \cdots s_{\ell}$ is a reduced expression then

$$
R_{L}(y)=\left\{s_{1} s_{2} \cdots s_{i} \cdots s_{2} s_{1} \mid 1 \leq i \leq \ell\right\} .
$$

Furthermore, the length $\ell(y)$ equals the number of reflection hyperplanes separating the alcoves $C_{\text {fund }}$ and $y \cdot C_{\text {fund }}$ by Theorem 4.5 in [Hum90. In fact, J. Humphreys shows that the set of reflection hyperplanes separating the alcoves $C_{\text {fund }}$ and $y \cdot C_{\text {fund }}$ 
equals $\left\{s_{1} s_{2} \cdots s_{i-1} \cdot H_{s_{i}} \mid 1 \leq i \leq \ell\right\}$. Combining these results, we obtain the following alternative description of $R_{L}(y)$ :

Lemma 2.1. For $y \in W_{\text {aff }}$, we have

$$
R_{L}(y)=\left\{s \in R\left(W_{\text {aff }}\right) \mid H_{s} \text { separates } C_{\text {fund }} \text { and } y \cdot C_{\text {fund }}\right\} .
$$

Proof. Fix a reduced expression $y=s_{1} \cdots s_{\ell}$. For $1 \leq i \leq \ell$, the hyperplane of fixed points of the reflection $s_{1} s_{2} \cdots s_{i} \cdots s_{2} s_{1}$ is $s_{1} s_{2} \cdots s_{i-1} \cdot H_{s_{i}}$ and the claim follows from the above discussion.

In order to prove the recursion formula and the duality formula, we will need three more lemmas about reflections in $W_{\text {aff }}$.

Lemma 2.2. Let $x \in W_{\text {aff }}$ and $s \in S$ such that $x<x s$. Then

$$
R_{L}(x s)=R_{L}(x) \sqcup\left\{x s x^{-1}\right\} .
$$

Proof. Let $x=s_{1} \cdots s_{\ell}$ be a reduced expression. The assumption implies that $x s=s_{1} \cdots s_{\ell} s$ is also a reduced expression and the claim follows from (2.1) as $x s x^{-1}=s_{1} s_{2} \cdots s_{\ell} s s_{\ell} \cdots s_{2} s_{1}$.

For Lemma 2.3, recall that $W_{\text {aff }}^{+}$denotes the set of elements $x \in W_{\text {aff }}$ whose length is minimal in the coset $W_{\text {fin }} x$.

Lemma 2.3. For $x, y \in W_{\text {aff }}^{+}$, there exists at most one reflection $s \in R_{L}(y)$ such that $s y \in W_{\text {fin }} x$.

Proof. Let $s, s^{\prime} \in R_{L}(y)$ such that $s y \in W_{\text {fin }} x$ and $s^{\prime} y \in W_{\text {fin }} x$. Then

$$
s s^{\prime}=\left(s y x^{-1}\right)\left(s^{\prime} y x^{-1}\right)^{-1} \in W_{\text {fin }},
$$

but $s \notin W_{\text {fin }}$ because $s y<y$ and $y$ has minimal length in the coset $W_{\text {fin }} y$. Hence we can write $s=s_{\beta, m}$ for some $m \neq 0$ and $\beta \in \Phi^{+}$and $s^{\prime}=s_{\beta^{\prime}, m^{\prime}}$ for some $m^{\prime} \in \mathbb{Z}$ and $\beta^{\prime} \in \Phi^{+}$. As $-\rho$ is fixed by all elements of $W_{\text {fin }}$ and as $s s^{\prime} \in W_{\text {fin }}$, we have

$$
p \cdot m \beta-\rho=s \cdot(-\rho)=s^{\prime} \cdot(-\rho)=p \cdot m^{\prime} \beta^{\prime}-\rho
$$

and therefore $m \beta=m^{\prime} \beta^{\prime}$. As $m \neq 0$, this implies that $\beta=\beta^{\prime}$ and $m=m^{\prime}$, so $s=s^{\prime}$.

Lemma 2.4. Let $x \in W_{\text {aff }}$ and $w \in W_{\text {fin }}$. Then

$$
w\left(R_{L}(x) \backslash R\left(W_{\text {fin }}\right)\right) w^{-1}=w R_{L}(x) w^{-1} \backslash R\left(W_{\text {fin }}\right)=R_{L}(w x) \backslash R\left(W_{\text {fin }}\right) .
$$

Proof. The first equality is clear since $R\left(W_{\text {fin }}\right)$ is stable under conjugation by elements of $W_{\text {fin. }}$. Now suppose that $s \in R_{L}(x)$ such that $w s w^{-1} \notin R\left(W_{\text {fin }}\right)$. By Lemma 2.1 the hyperplane $H_{s}$ separates the alcoves $C_{\text {fund }}$ and $x \cdot C_{\text {fund }}$ and it follows that $w \cdot H_{s}=H_{w s w^{-1}}$ separates $w \cdot C_{\text {fund }}$ and $w x \cdot C_{\text {fund }}$. However, $H_{w s w^{-1}}$ does not separate $C_{\text {fund }}$ and $w \cdot C_{\text {fund }}$. Indeed, the point $-\rho$ is contained in the intersection of the closures of the alcoves $C_{\text {fund }}$ and $w \cdot C_{\text {fund }}$, so any hyperplane separating these alcoves must contain $-\rho$ and therefore correspond to a reflection in $W_{\text {fin }}$. Hence $C_{\text {fund }}$ and $w \cdot C_{\text {fund }}$ belong to the same connected component of $X_{\mathbb{R}} \backslash H_{w s w^{-1}}$ and $w x \cdot C_{\text {fund }}$ belongs to the other one. Using Lemma 2.1 again, we conclude that $w s w^{-1} \in R_{L}(w x) \backslash R\left(W_{\text {fin }}\right)$, so

$$
w R_{L}(x) w^{-1} \backslash R\left(W_{\text {fin }}\right) \subseteq R_{L}(w x) \backslash R\left(W_{\text {fin }}\right) .
$$


Conversely, if $s \in R_{L}(w x) \backslash R\left(W_{\text {fin }}\right)$ then

$w^{-1} s w \in w^{-1}\left(R_{L}(w x) \backslash R\left(W_{\text {fin }}\right)\right) w=w^{-1} R_{L}(w x) w \backslash R\left(W_{\text {fin }}\right) \subseteq R_{L}(x) \backslash R\left(W_{\text {fin }}\right)$

and it follows that $s \in w\left(R_{L}(x) \backslash R\left(W_{\text {fin }}\right)\right) w^{-1}$. We conclude that

$$
R_{L}(w x) \backslash R\left(W_{\text {fin }}\right) \subseteq w\left(R_{L}(x) \backslash R\left(W_{\text {fin }}\right)\right) w^{-1}=w R_{L}(x) w^{-1} \backslash R\left(W_{\text {fin }}\right),
$$

as required.

Now let $h=\left\langle\rho, \alpha_{\mathrm{h}}^{\vee}\right\rangle+1$ be the Coxeter number of $G$. We conclude this section with a lemma about $W_{\text {aff }}^{+}$that is well known to experts.

Lemma 2.5. Suppose that $p \geq h$. Then

$$
W_{\text {aff }}^{+}=\left\{x \in W_{\text {aff }} \mid x \cdot C_{\text {fund }} \cap X^{+} \neq \varnothing\right\} .
$$

Proof. First note that $\alpha_{\mathrm{h}}^{\vee}$ is the highest root in the dual root system $\Phi^{\vee}$, hence $\left\langle\rho, \beta^{\vee}\right\rangle \leq\left\langle\rho, \alpha_{\mathrm{h}}^{\vee}\right\rangle<p$ for all $\beta \in \Phi^{+}$. This implies that $0 \in C_{\text {fund }}$ and that $x \cdot C_{\text {fund }} \cap X \neq \varnothing$ for all $x \in W_{\text {aff }}$.

Now let $X_{\mathbb{R}}^{+}=\left\{\lambda \in X_{\mathbb{R}} \mid\left\langle\lambda, \beta^{\vee}\right\rangle>0\right.$ for all $\left.\beta \in \Phi^{+}\right\}$be the dominant Weyl chamber in $X_{\mathbb{R}}$. As the finite Weyl group $W_{\text {fin }}$ acts simply transitively on the set of Weyl chambers, every coset $W_{\text {fin }} x$ contains a unique element $y$ with $y \cdot C_{\text {fund }} \subseteq$ $X_{\mathbb{R}}^{+}-\rho$ and we claim that $y$ has minimal length among the elements of $W_{\text {fin }} x$. Indeed, it is straightforward to see that an alcove $C$ satisfies $C \subseteq X_{\mathbb{R}}^{+}-\rho$ if and only if none of the reflection hyperplanes $H_{\beta, 0}^{p}$ with $\beta \in \Phi^{+}$separate $C_{\text {fund }}$ and $C$, so Lemma 2.2 implies that $y$ is the unique element of $W_{\text {fin }} x$ with $R_{L}(y) \cap R\left(W_{\text {fin }}\right)=\varnothing$. As $\ell(y)=\left|R_{L}(y)\right|$, the claim follows from Lemma 2.4.

For $y \in W_{\text {aff }}$ with $y \cdot C_{\text {fund }} \subseteq X_{\mathbb{R}}^{+}-\rho$ and for $\lambda \in y \cdot C_{\text {fund }} \cap X$, we have $0\left\langle\left\langle\lambda+\rho, \alpha^{\vee}\right\rangle=\left\langle\lambda, \alpha^{\vee}\right\rangle+1\right.$ for all $\alpha \in \Delta$ and it follows that $\lambda \in X^{+}$. Conversely, if $y \cdot C_{\text {fund }}$ contains a dominant weight then $y \cdot C_{\text {fund }} \cap\left(X_{\mathbb{R}}^{+}-\rho\right) \neq \varnothing$ and therefore $y \cdot C_{\text {fund }} \subseteq X_{\mathbb{R}}-\rho$ by connectedness of $y \cdot C_{\text {fund }}$. We conclude that an alcove $x \cdot C_{\text {fund }}$ contains a dominant weight if and only if $x$ has minimal length among the elements of $W_{\text {fin }} x$, as required.

\section{REWRITING THE SUM FORMULAS}

Suppose that $p \geq h$, the Coxeter number of $G$. In this section, we rewrite the sum formulas of Jantzen and Andersen in a character- and dimension-free way by interpreting them as objects of the anti-spherical $W_{\text {aff-module and its dual, }}$ respectively.

Let $\lambda \in X^{+}$be $p$-regular and let $x \in W_{\text {aff }}^{+}$and $\lambda_{0} \in C_{\text {fund }}$ such that $\lambda=x \cdot \lambda_{0}$. Recall that

$$
\begin{aligned}
\mathrm{JSF}_{\lambda} & =-\sum_{\alpha \in \Phi^{+}} \sum_{0<m<\left\langle\lambda+\rho, \alpha^{\vee}\right\rangle} \nu_{p}(m) \cdot \chi_{\lambda-m \alpha} \\
& =-\sum_{\alpha \in \Phi^{+}} \sum_{0<m p<\left\langle\lambda+\rho, \alpha^{\vee}\right\rangle} \nu_{p}(m p) \cdot \chi_{\lambda-m p \alpha}
\end{aligned}
$$

and note that for $\alpha \in \Phi^{+}$and $m \in \mathbb{Z}$, the condition that $0<m p\left\langle\left\langle\lambda+\rho, \alpha^{\vee}\right\rangle\right.$ is satisfied if and only if the reflection hyperplane $H_{\alpha, m}^{p}$ separates the alcoves $C_{\text {fund }}$ and $x \cdot C_{\text {fund }}$. Furthermore, we have

$$
\lambda-m p \alpha=t_{-m \alpha} \cdot \lambda=s_{\alpha} t_{m \alpha} s_{\alpha} \cdot \lambda=s_{\alpha} \cdot\left(s_{\alpha, m} \cdot \lambda\right)
$$


and so $\chi_{\lambda-m p \alpha}=-\chi_{s_{\alpha, m} \cdot \lambda}$. Combining these observations with Lemma 2.1, we obtain

$$
\mathrm{JSF}_{x \cdot \lambda_{0}}=\sum_{s \in R_{L}(x)} \nu_{p}(m(s) \cdot p) \cdot \chi_{s x} \cdot \lambda_{0}
$$

where for $s=s_{\alpha, m}$, we set $m(s):=m$.

Next, we wish to rewrite the formula (3.1) in terms of the Coxeter combinatorics associated with the affine Weyl group. Let $M_{\text {asph }}:=\operatorname{sign} \otimes_{\mathbb{Z}\left[W_{\text {fin }}\right]} \mathbb{Z}\left[W_{\text {aff }}\right]$ be the

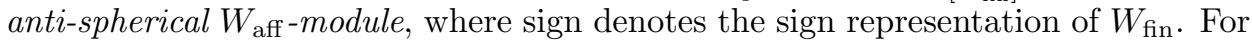
$x \in W_{\text {aff }}$, we write $N_{x}:=1 \otimes x \in M_{\text {asph }}$, so that the elements $N_{y}$ with $y \in W_{\text {aff }}^{+}$ form a $\mathbb{Z}$-basis of $M_{\text {asph. }}$. Note that for $w \in W_{\text {fin }}$ and $x, y \in W_{\text {aff }}$, we have

$$
N_{w x}=\operatorname{det}(w) \cdot N_{x} \quad \text { and } \quad N_{x y}=N_{x} \cdot y .
$$

Now fix $\lambda_{0} \in C_{\text {fund }} \cap X$ and recall that the characters $\chi_{x} \cdot \lambda_{0}$ with $x \in W_{\text {aff }}^{+}$form a basis of the $\mathbb{Z}$-submodule $\mathbb{Z}[X]_{\lambda_{0}}^{W_{\text {fin }}}$ of $\mathbb{Z}[X]^{W_{\text {fin }}}$ spanned by the characters of all $G$-modules in $\operatorname{Rep}_{\lambda_{0}}(G)$. There is an isomorphism of $\mathbb{Z}$-modules

$$
\psi_{\lambda_{0}}: \mathbb{Z}[X]_{\lambda_{0}}^{W_{\text {fin }}} \longrightarrow M_{\text {asph }}
$$

with $\chi_{y \cdot \lambda_{0}} \mapsto N_{y}$ for all $y \in W_{\text {aff }}^{+}$. (This can be upgraded to an isomorphism of $\mathbb{Z}\left[W_{\text {aff }}\right]$-modules by identifying $\mathbb{Z}[X]_{\lambda_{0}}^{W_{\text {fin }}}$ with the Grothendieck group of $\operatorname{Rep}_{\lambda_{0}}(G)$ and letting $s+1$ act via a wall-crossing functor $\Theta_{s}$ for all $s \in S$.) For $x \in W_{\text {aff }}^{+}$, we define

$$
\mathrm{JSF}_{x}:=\psi_{\lambda_{0}}\left(\mathrm{JSF}_{x \cdot \lambda_{0}}\right)
$$

Lemma 3.1. For all $x \in W_{\text {aff }}$, we have $\psi_{\lambda_{0}}\left(\chi_{x \cdot \lambda_{0}}\right)=N_{x}$.

Proof. For $x \in W_{\text {aff }}^{+}$, this is just the definition of $\psi_{\lambda_{0}}$. For an arbitrary $x \in W_{\text {aff }}$, we can write $x=w y$ with $w \in W_{\text {fin }}$ and $y \in W_{\text {aff }}^{+}$and it follows that

$$
\psi_{\lambda_{0}}\left(\chi_{x} \cdot \lambda_{0}\right)=\psi_{\lambda_{0}}\left(\operatorname{det}(w) \cdot \chi_{y \cdot \lambda_{0}}\right)=\operatorname{det}(w) \cdot N_{y}=N_{x},
$$

as claimed.

Corollary 3.2. For $x \in W_{\text {aff }}^{+}$, we have

$$
\mathrm{JSF}_{x}=\sum_{\substack{s \in R\left(W_{\mathrm{aff}}\right) \\ s x<x}} \nu_{p}(m(s) \cdot p) \cdot N_{s x} .
$$

Proof. This is immediate from equation (3.1) and Lemma 3.1 .

Remark 3.3. Fix a reduced expression $x=s_{1} \cdots s_{\ell}$ for $x \in W_{\text {aff }}^{+}$and set

$$
t_{i}=s_{1} s_{2} \cdots s_{i} \cdots s_{2} s_{1} \quad \text { and } \quad x_{i}=t_{i} x=s_{1} \cdots s_{i-1} s_{i+1} \cdots s_{\ell}
$$

for $1 \leq i \leq \ell$. Then $R_{L}(x)=\left\{t_{i} \mid 1 \leq i \leq \ell\right\}$ by (2.1) and

$$
\mathrm{JSF}_{x}=\sum_{i=1}^{\ell} \nu_{p}\left(m\left(t_{i}\right) \cdot p\right) \cdot N_{x_{i}}
$$

by Corollary 3.2 . 
In order to rewrite the Andersen sum formula, we work in the dual $M_{\text {asph }}^{*}=$ $\operatorname{Hom}_{\mathbb{Z}}\left(M_{\mathrm{asph}}, \mathbb{Z}\right)$ of the anti-spherical module $M_{\text {asph }}$. We write

$$
\langle-,-\rangle: M_{\text {asph }}^{*} \times M_{\text {asph }} \longrightarrow \mathbb{Z}
$$

for the natural evaluation pairing. For $x \in W_{\text {aff }}^{+}$, we define an element $N_{x}^{*} \in M_{\text {asph }}^{*}$ by $\left\langle N_{x}^{*}, N_{y}\right\rangle=\delta_{x, y}$ for all $y \in W_{\text {aff }}^{+}$. Then every element $\vartheta \in M_{\text {asph }}^{*}$ can be written as a formal infinite sum $\vartheta=\sum_{x} a_{x} \cdot N_{x}^{*}$ with $a_{x} \in \mathbb{Z}$ for all $x \in W_{\text {aff }}^{+}$. Note that we do not require that all but finitely many of the $a_{x}$ are zero, the infinite sum is to be understood in the sense that $\left\langle\vartheta, N_{x}\right\rangle=a_{x}$ for all $x \in W_{\text {aff }}^{+}$. For $y \in W_{\text {aff }}$, there exist unique elements $w \in W_{\text {fin }}$ and $x \in W_{\text {aff }}^{+}$with $y=w x$ and we set $N_{y}^{*}:=\operatorname{det}(w) \cdot N_{x}^{*}$.

Now let $\lambda \in X^{+}$be $p$-regular and let $x \in W_{\text {aff }}^{+}$and $\lambda_{0} \in C_{\text {fund }}$ such that $\lambda=x \cdot \lambda_{0}$. Recall that

$$
\begin{aligned}
\operatorname{ASF}(\lambda, T)= & -\sum_{\alpha \in \Phi^{+}} \sum_{m \notin I(\lambda, \alpha)} \nu_{p}(m) \cdot\left[\operatorname{ch} T: \chi_{\lambda-m \alpha}\right] \\
& =-\sum_{\alpha \in \Phi^{+}} \sum_{m p \notin I(\lambda, \alpha)} \nu_{p}(m p) \cdot\left[\operatorname{ch} T: \chi_{\lambda-m p \alpha}\right],
\end{aligned}
$$

where $I(\lambda, \alpha)=\left\{m \in \mathbb{Z} \mid 0 \leq m \leq\left\langle\lambda+\rho, \alpha^{\vee}\right\rangle\right\}$. For $\alpha \in \Phi^{+}$and $m \in \mathbb{Z}$, the condition that $m p \notin I(\lambda, \alpha)$ is satisfied if and only if $m \neq 0$ and the hyperplane $H_{\alpha, m}^{p}$ does not separate the alcoves $C_{\text {fund }}$ and $x \cdot C_{\text {fund }}$. As before, we have

$$
\left[\operatorname{ch} T: \chi_{\lambda-m p \alpha}\right]=-\left[\operatorname{ch} T: \chi_{s_{\alpha, m} \cdot \lambda}\right],
$$

and using Lemma 2.1 we can rewrite the Andersen sum formula as

$$
\operatorname{ASF}\left(x \cdot \lambda_{0}, T\right)=\sum_{\substack{s \in R\left(W_{\text {aff }}\right) \backslash R\left(W_{\text {fin }}\right) \\ s x>x}} \nu_{p}(m(s) \cdot p) \cdot\left[\operatorname{ch} T: \chi_{s x} \cdot \lambda_{0}\right] .
$$

Now the characters of the indecomposable tilting modules in $\operatorname{Rep}_{\lambda_{0}}(G)$ form a basis of $\mathbb{Z}[X]_{\lambda_{0}}^{W_{\text {fin }}}$, so the Andersen sum formula defines a $\mathbb{Z}$-linear map

$$
\mathrm{ASF}_{\lambda}: \mathbb{Z}[X]_{\lambda_{0}}^{W_{\text {fin }}} \longrightarrow \mathbb{Z}
$$

with $\left\langle\operatorname{ASF}_{\lambda}, \operatorname{ch} T\right\rangle=\operatorname{ASF}(\lambda, T)$ for every tilting module $T$ in $\operatorname{Rep}_{\lambda_{0}}(G)$, where as before, we write

$$
\langle-,-\rangle:\left(\mathbb{Z}[X]_{\lambda_{0}}^{W_{\text {fin }}}\right)^{*} \times \mathbb{Z}[X]_{\lambda_{0}}^{W_{\text {fin }}} \longrightarrow \mathbb{Z}
$$

for the evaluation pairing. The isomorphism $\psi_{\lambda_{0}}: \mathbb{Z}[X]_{\lambda_{0}}^{W_{\text {fin }}} \rightarrow M_{\text {asph }}$ induces an isomorphism

$$
\psi_{\lambda_{0}}^{*}: M_{\text {asph }}^{*} \longrightarrow\left(\mathbb{Z}[X]_{\lambda_{0}}^{W_{\text {fin }}}\right)^{*}
$$

and we define $\operatorname{ASF}_{x} \in M_{\text {asph }}^{*}$ by the equality

$$
\psi_{\lambda_{0}}^{*}\left(\mathrm{ASF}_{x}\right)=\mathrm{ASF}_{x \cdot \lambda_{0}} .
$$

Lemma 3.4. For all $y \in W_{\text {aff }}$, the $\mathbb{Z}$-linear map $\left[-: \chi_{y \cdot \lambda_{0}}\right]: \mathbb{Z}[X]_{\lambda_{0}}^{W_{\text {fin }}} \rightarrow \mathbb{Z}$ with $\chi \mapsto\left[\chi: \chi_{y \cdot \lambda_{0}}\right]$ satisfies $\left[-: \chi_{y \cdot \lambda_{0}}\right]=\psi_{\lambda_{0}}^{*}\left(N_{y}^{*}\right)$.

Proof. Let $w \in W_{\text {fin }}$ and $x \in W_{\text {aff }}^{+}$such that $y=w x$. For $z \in W_{\text {aff }}^{+}$, we have

$$
\begin{aligned}
{\left[\chi_{z \cdot \lambda_{0}}: \chi_{y \cdot \lambda_{0}}\right]=\operatorname{det}(w) \cdot \delta_{x, z} } & =\operatorname{det}(w) \cdot\left\langle N_{x}^{*}, N_{z}\right\rangle=\left\langle N_{y}^{*}, N_{z}\right\rangle \\
& =\left\langle N_{y}^{*}, \psi_{\lambda_{0}}\left(\chi_{z \cdot \lambda_{0}}\right)\right\rangle=\left\langle\psi_{\lambda_{0}}^{*}\left(N_{y}^{*}\right), \chi_{z \cdot \lambda_{0}}\right\rangle
\end{aligned}
$$

and the claim follows. 
Corollary 3.5. For $x \in W_{\text {aff }}^{+}$, we have

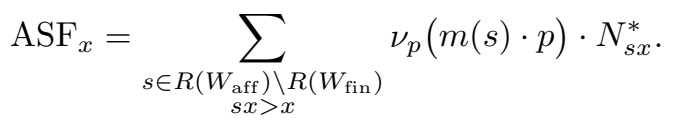

Proof. By Lemma 3.4 and equation (3.2), we have

$$
\begin{aligned}
& \psi_{\lambda_{0}}^{*}\left(\sum_{s \in R\left(W_{\text {aff }}\right) \backslash R\left(W_{\text {fin }}\right)} \nu_{p}(m(s) \cdot p) \cdot N_{s x}^{*}\right)
\end{aligned}
$$

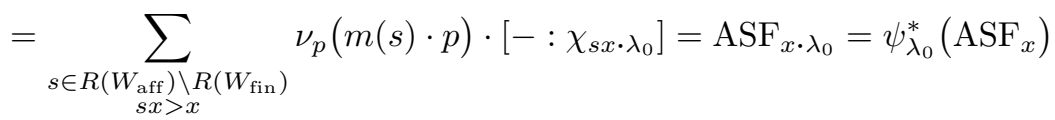

and the claim follows.

4. The RECURSion FORMula AND the DuAlity FORMula

In this section, we prove the two formulas that were announced in the introduction. We start with the recursion formula.

Theorem 4.1 (Recursion formula). Let $x \in W_{\mathrm{aff}}^{+}$and $s \in S$ with $x<x s \in W_{\mathrm{aff}}^{+}$. Then

$$
\mathrm{JSF}_{x s}=\nu_{p}\left(m\left(x s x^{-1}\right) \cdot p\right) \cdot N_{x}+\mathrm{JSF}_{x} \cdot s .
$$

Proof. Recall from Corollary 3.2 that

$$
\mathrm{JSF}_{x}=\sum_{t \in R_{L}(x)} \nu_{p}(m(t) \cdot p) \cdot N_{t x} \quad \text { and } \quad \mathrm{JSF}_{x s}=\sum_{t \in R_{L}(x s)} \nu_{p}(m(t) \cdot p) \cdot N_{t x s},
$$

where $R_{L}(x s)=R_{L}(x) \sqcup\left\{x s x^{-1}\right\}$ by Lemma 2.1. We conclude that

$$
\begin{aligned}
\mathrm{JSF}_{x s} & =\sum_{t \in R_{L}(x s)} \nu_{p}(m(t) \cdot p) \cdot N_{t x s} \\
& =\nu_{p}\left(m\left(x s x^{-1}\right) \cdot p\right) \cdot N_{x}+\sum_{t \in R_{L}(x)} \nu_{p}(m(t) \cdot p) \cdot N_{t x s} \\
& =\nu_{p}\left(m\left(x s x^{-1}\right) \cdot p\right) \cdot N_{x}+\sum_{t \in R_{L}(x)} \nu_{p}(m(t) \cdot p) \cdot N_{t x} \cdot s \\
& =\nu_{p}\left(m\left(x s x^{-1}\right) \cdot p\right) \cdot N_{x}+\mathrm{JSF}_{x} \cdot s,
\end{aligned}
$$

as claimed.

Next we prove the duality formula, which shows that the Jantzen filtration and the Andersen filtration are closely related to each other, at least on a combinatorial level.

Theorem 4.2 (Duality formula). For all $x, y \in W_{\text {aff }}^{+}$, we have

$$
\left\langle\mathrm{ASF}_{x}, N_{y}\right\rangle=\left\langle N_{x}^{*}, \mathrm{JSF}_{y}\right\rangle .
$$

Proof. By Corollaries 3.2 and 3.5. we have

$$
\mathrm{JSF}_{y}=\sum_{\substack{s \in R\left(W_{\text {aff }}\right) \\ s y<y}} \nu_{p}(m(s) \cdot p) \cdot N_{s y}
$$


and

$$
\operatorname{ASF}_{x}=\sum_{\substack{s \in R\left(W_{\mathrm{aff}}\right) \backslash R\left(W_{\mathrm{fin}}\right) \\ s x>x}} \nu_{p}(m(s) \cdot p) \cdot N_{s x}^{*} .
$$

If we write $\mathrm{JSF}_{y}=\sum_{z} a_{y, z} \cdot N_{z}$ in terms of the basis $\left\{N_{z} \mid z \in W_{\text {aff }}^{+}\right\}$then a summand $\nu_{p}(m(s) \cdot p) \cdot N_{s y}$ with $s \in R_{L}(y)$ contributes $\operatorname{det}(w) \cdot \nu_{p}(m(s) \cdot p)$ to $a_{y, z}$ precisely when $s y=w z$ for some $w \in W_{\text {fin }}$. Furthermore, for any $z \in W_{\text {aff }}^{+}$, there exists at most one $s \in R_{L}(y)$ with $s y \in W_{\text {fin }} z$ by Lemma 2.3. We conclude that

$$
a_{y, z}= \begin{cases}\operatorname{det}(w) \cdot \nu_{p}(m(s) \cdot p) & \text { if } s y=w z \text { for some } s \in R_{L}(y) \text { and } w \in W_{\text {fin }}, \\ 0 & \text { otherwise. }\end{cases}
$$

Analogously, we can write $\mathrm{ASF}_{x}=\sum_{z} b_{x, z} \cdot N_{z}^{*}$ with

$$
b_{x, z}= \begin{cases}\operatorname{det}(w) \cdot \nu_{p}(m(s) \cdot p) & \text { if } x<s x=w z \text { for some } s \in R\left(W_{\mathrm{aff}}\right) \backslash R\left(W_{\mathrm{fin}}\right) \\ 0 & \text { and } w \in W_{\mathrm{fin}}, \\ & \text { otherwise. }\end{cases}
$$

If $s y=w x$ for some $s \in R_{L}(y)$ and $w \in W_{\text {fin }}$ then $s \notin W_{\text {fin }}$ because $s y<y$ and $y$ is minimal in the coset $W_{\text {fin }} y$. By Lemma 2.4, we have $w^{-1} s w \in R_{L}\left(w^{-1} y\right) \backslash R\left(W_{\text {fin }}\right)$ and it follows that

$$
x=\left(w^{-1} s w\right) w^{-1} y<w^{-1} y=\left(w^{-1} s w\right) x .
$$

This implies that

$$
a_{y, x}=\operatorname{det}(w) \cdot \nu_{p}(m(s) \cdot p)=\operatorname{det}\left(w^{-1}\right) \cdot \nu_{p}\left(m\left(w^{-1} s w\right) \cdot p\right)=b_{x, y} .
$$

If $x<s x=w y$ for some $s \in R\left(W_{\text {aff }}\right) \backslash R\left(W_{\text {fin }}\right)$ and $w \in W_{\text {fin }}$ then $s \in R_{L}(w y)$ and $w^{-1} s w \in R_{L}(y)$ by Lemma 2.4. Furthermore, we have $w^{-1} x=\left(w^{-1} s w\right) y$ and it follows that

$$
b_{x, y}=\operatorname{det}(w) \cdot \nu_{p}(m(s) \cdot p)=\operatorname{det}\left(w^{-1}\right) \cdot \nu_{p}\left(m\left(w^{-1} s w\right) \cdot p\right)=a_{y, x} .
$$

We conclude that

$$
\left\langle\mathrm{ASF}_{x}, N_{y}\right\rangle=b_{x, y}=a_{y, x}=\left\langle N_{x}^{*}, \mathrm{JSF}_{y}\right\rangle
$$

for all $x, y \in W_{\text {aff }}^{+}$.

Remark 4.3. The duality formula

$$
\left\langle\mathrm{ASF}_{x}, N_{y}\right\rangle=\left\langle N_{x}^{*}, \mathrm{JSF}_{y}\right\rangle
$$

can be generalized to non-regular weights, even when the characteristic $p$ is smaller than the Coxeter number of $G$. For $\lambda \in X^{+}$, we define $\operatorname{JSF}_{\lambda} \in \mathbb{Z}[X]^{W_{\text {fin }}}$ and $\mathrm{ASF}_{\lambda} \in\left(\mathbb{Z}[X]^{W_{\text {fin }}}\right)^{*}$ by

$$
\mathrm{JSF}_{\lambda}=\sum_{i>0} \operatorname{ch} \Delta(\lambda)^{i} \quad \text { and } \quad\left\langle\mathrm{ASF}_{\lambda}, \operatorname{ch} T\right\rangle=\sum_{i>0} \operatorname{dim} \bar{F}_{\lambda}^{i}(T)
$$

for every tilting module $T$, where $\langle-,-\rangle:\left(\mathbb{Z}[X]^{W_{\text {fin }}}\right)^{*} \times \mathbb{Z}[X]^{W_{\text {fin }}} \rightarrow \mathbb{Z}$ denotes the evaluation pairing. Writing $\chi_{\lambda}^{*}=\left[-: \chi_{\lambda}\right] \in\left(\mathbb{Z}[X]^{W_{\text {fin }}}\right)^{*}$ for the 'dual basis' with $\left\langle\chi_{\lambda}^{*}, \chi_{\mu}\right\rangle=\delta_{\lambda, \mu}$ for all $\mu \in X^{+}$, we have the following natural extension of the duality formula to arbitrary weights:

$$
\left\langle\mathrm{ASF}_{\lambda}, \chi_{\mu}\right\rangle=\left\langle\chi_{\lambda}^{*}, \mathrm{JSF}_{\mu}\right\rangle .
$$


This formula has already appeared (implicitly) in AK08. (Compare the bijection constructed in the proof of Proposition 4.9 in AK08 with the sum formulas from Section 1.) In the same paper, H. H. Andersen and U. Kulkarni also give a representation theoretic explanation using a certain torsion Euler characteristic, see also Section 5.3 .

\section{TWO INTERPRETATIONS OF THE RECURSION FORMULA}

In this section, we give two representation theoretic explanations for the recursion formula

$$
\mathrm{JSF}_{x s}=\nu_{p}\left(x s x^{-1}\right) \cdot N_{x}+\mathrm{JSF}_{x} \cdot s
$$

from Theorem 4.1. In both cases, we use wall-crossing functors to relate the sum formulas for Weyl modules with highest weights in adjacent alcoves.

Recall that we write $G_{\mathbb{Z}_{p}}$ for the group scheme over $\mathbb{Z}_{p}$ corresponding to $G$. As the Jantzen filtration of the Weyl module $\Delta(\lambda)$ is defined using the generator $c_{\lambda}$ of $\operatorname{Hom}_{G_{\mathbb{Z}_{p}}}\left(\Delta_{\mathbb{Z}_{p}}(\lambda), \nabla_{\mathbb{Z}_{p}}(\lambda)\right)$, we work with lifts of translation functors to the category $\operatorname{Rep}\left(G_{\mathbb{Z}_{p}}\right)$ of finitely generated rational $G_{\mathbb{Z}_{p}}$-modules. This is possible by results of H. H. Andersen, as explained below.

5.1. Translation functors over $\mathbb{Z}_{p}$. By Proposition 5.2 and Theorem 5.4 in And83, the category $\operatorname{Rep}\left(G_{\mathbb{Z}_{p}}\right)$ admits a decomposition into linkage classes

$$
\operatorname{Rep}\left(G_{\mathbb{Z}_{p}}\right)=\bigoplus_{\lambda \in \bar{C}_{\text {fund }} \cap X} \operatorname{Rep}_{\lambda}\left(G_{\mathbb{Z}_{p}}\right),
$$

where $\operatorname{Rep}_{\lambda}\left(G_{\mathbb{Z}_{p}}\right)$ denotes the full subcategory of $G_{\mathbb{Z}_{p}}$-modules $M$ such that $M \otimes \mathbb{F}$ belongs to $\operatorname{Rep}_{\lambda}(G)$. Using the projection functors $\operatorname{pr}_{\lambda}: \operatorname{Rep}\left(G_{\mathbb{Z}_{p}}\right) \rightarrow \operatorname{Rep}_{\lambda}\left(G_{\mathbb{Z}_{p}}\right)$, it is possible to define mutually right and left adjoint exact translation functors

$$
T_{\lambda}^{\mu}: \operatorname{Rep}_{\lambda}\left(G_{\mathbb{Z}_{p}}\right) \rightarrow \operatorname{Rep}_{\mu}\left(G_{\mathbb{Z}_{p}}\right) \quad \text { and } \quad T_{\mu}^{\lambda}: \operatorname{Rep}_{\mu}\left(G_{\mathbb{Z}_{p}}\right) \rightarrow \operatorname{Rep}_{\lambda}\left(G_{\mathbb{Z}_{p}}\right)
$$

for $\lambda, \mu \in \bar{C}_{\text {fund }} \cap X$ just as in Section II.7 in [Jan03, see Lemma 5.5 in [And83]. For $G_{\mathbb{Z}_{p}}$-modules $M$ and $N$ in $\operatorname{Rep}_{\lambda}\left(G_{\mathbb{Z}_{p}}\right)$ and $\operatorname{Rep}_{\mu}\left(G_{\mathbb{Z}_{p}}\right)$, respectively, the adjunction between $T_{\lambda}^{\mu}$ and $T_{\mu}^{\lambda}$ gives rise to natural isomorphisms

and

$$
\operatorname{adj}_{1}: \operatorname{Hom}_{G_{\mathbb{Z}_{p}}}\left(M, T_{\mu}^{\lambda} N\right) \longrightarrow \operatorname{Hom}_{G_{\mathbb{Z}_{p}}}\left(T_{\lambda}^{\mu} M, N\right)
$$

such that

$$
\operatorname{adj}_{2}: \operatorname{Hom}_{G_{\mathbb{Z}_{p}}}\left(N, T_{\lambda}^{\mu} M\right) \longrightarrow \operatorname{Hom}_{G_{\mathbb{Z}_{p}}}\left(T_{\mu}^{\lambda} N, M\right)
$$

$$
\begin{aligned}
& \operatorname{adj}_{1}(f \circ g)=\operatorname{adj}_{1}(f) \circ T_{\lambda}^{\mu}(g), \quad \operatorname{adj}_{2}(f \circ g)=\operatorname{adj}_{2}(f) \circ T_{\mu}^{\lambda}(g), \\
& \operatorname{adj}_{1}\left(T_{\mu}^{\lambda} f \circ g\right)=f \circ \operatorname{adj}_{1}(g),
\end{aligned}
$$

for all morphisms $f$ and $g$ in suitable Hom-spaces. Now fix $\lambda \in C_{\text {fund }} \cap X$ and $\mu \in \bar{C}_{\text {fund }} \cap X$ such that $\operatorname{Stab}_{W_{\text {aff }}}(\mu)=\{1, s\}$ for some $s \in S$. For $x \in W_{\text {aff }}^{+}$with $x<x s$ and $x s \in W_{\text {aff }}^{+}$, we have $x \cdot \mu \in X^{+}$and there are isomorphisms

$$
T_{\lambda}^{\mu} \Delta_{\mathbb{Z}_{p}}(x \cdot \lambda) \cong \Delta_{\mathbb{Z}_{p}}(x \cdot \mu) \cong T_{\lambda}^{\mu} \Delta_{\mathbb{Z}_{p}}(x s \cdot \lambda)
$$

and

$$
T_{\lambda}^{\mu} \nabla_{\mathbb{Z}_{p}}(x \cdot \lambda) \cong \nabla_{\mathbb{Z}_{p}}(x \cdot \mu) \cong T_{\lambda}^{\mu} \nabla_{\mathbb{Z}_{p}}(x s \cdot \lambda) .
$$

Furthermore, there are short exact sequences

$$
0 \longrightarrow \Delta_{\mathbb{Z}_{p}}(x s \cdot \lambda) \stackrel{i}{\longrightarrow} T_{\mu}^{\lambda} \Delta_{\mathbb{Z}_{p}}(x \cdot \mu) \stackrel{\pi}{\longrightarrow} \Delta_{\mathbb{Z}_{p}}(x \cdot \lambda) \longrightarrow 0
$$


and

$$
0 \longrightarrow \nabla_{\mathbb{Z}_{p}}(x \cdot \lambda) \stackrel{i^{\prime}}{\longrightarrow} T_{\mu}^{\lambda} \nabla_{\mathbb{Z}_{p}}(x \cdot \mu) \stackrel{\pi^{\prime}}{\longrightarrow} \nabla_{\mathbb{Z}_{p}}(x s \cdot \lambda) \longrightarrow 0
$$

with

and

$$
i=\operatorname{adj}_{1}^{-1}\left(\operatorname{id}_{\Delta_{\mathbb{Z}_{p}}(x \cdot \mu)}\right), \quad i^{\prime}=\operatorname{adj}_{1}^{-1}\left(\operatorname{id}_{\nabla_{\mathbb{Z}_{p}}(x \cdot \mu)}\right)
$$

$$
\pi=\operatorname{adj}_{2}\left(\operatorname{id}_{\Delta_{\mathbb{Z}_{p}}(x \cdot \mu)}\right), \quad \pi^{\prime}=\operatorname{adj}_{2}\left(\operatorname{id}_{\nabla_{\mathbb{Z}_{p}}(x \cdot \mu)}\right),
$$

see Section 2.4 in And97. Let us further consider the morphisms

$$
\begin{gathered}
r=\operatorname{adj}_{2}\left(\operatorname{id}_{\Delta_{\mathbb{Z}_{p}}(x \cdot \mu)}\right): T_{\mu}^{\lambda} \Delta_{\mathbb{Z}_{p}}(x \cdot \mu) \longrightarrow \Delta_{\mathbb{Z}_{p}}(x s \cdot \lambda) \\
r^{\prime}=\operatorname{adj}_{2}\left(\operatorname{id}_{\nabla_{\mathbb{Z}_{p}}(x \cdot \mu)}\right): T_{\mu}^{\lambda} \nabla_{\mathbb{Z}_{p}}(x \cdot \mu) \longrightarrow \nabla_{\mathbb{Z}_{p}}(x \cdot \lambda) \\
s=\operatorname{adj}_{1}^{-1}\left(\operatorname{id}_{\Delta_{\mathbb{Z}_{p}}(x \cdot \mu)}\right): \Delta_{\mathbb{Z}_{p}}(x \cdot \lambda) \longrightarrow T_{\mu}^{\lambda} \Delta_{\mathbb{Z}_{p}}(x \cdot \mu) \\
s^{\prime}=\operatorname{adj}_{1}^{-1}\left(\operatorname{id}_{\nabla_{\mathbb{Z}_{p}}(x \cdot \mu)}\right): \nabla_{\mathbb{Z}_{p}}(x s \cdot \lambda) \longrightarrow T_{\mu}^{\lambda} \nabla_{\mathbb{Z}_{p}}(x \cdot \mu) .
\end{gathered}
$$

The following two lemmas give certain relations for the composition of these homomorphisms and for their composition with the homomorphisms $c_{x \cdot \lambda}$ and $c_{x s \cdot \lambda}$.

Lemma 5.1 (Andersen). Let $m=\nu_{p}(\operatorname{dim} \Delta(x \cdot \mu))$. Then we have

$$
\begin{array}{ll}
r \circ i=p^{m} \cdot \operatorname{id}_{\Delta_{\mathbb{Z}_{p}}(x s \cdot \lambda)}, & r^{\prime} \circ i^{\prime}=p^{m} \cdot \operatorname{id}_{\nabla_{\mathbb{Z}_{p}}(x \cdot \lambda),}, \\
\pi \circ s=p^{m} \cdot \operatorname{id}_{\Delta_{\mathbb{Z}_{p}}(x \cdot \lambda)}, & \pi^{\prime} \circ s^{\prime}=p^{m} \cdot \operatorname{id}_{\nabla_{\mathbb{Z}_{p}}(x s \cdot \lambda) .} .
\end{array}
$$

Proof. See Lemma 2.4 in And97.

Lemma 5.2 (Andersen). Let $\beta=T_{\mu}^{\lambda} c_{x \cdot \mu}$. Up to units in $\mathbb{Z}_{p}$, we have $c_{x \cdot \mu}=T_{\lambda}^{\mu} c_{x \cdot \lambda}$ and there are commutative diagrams

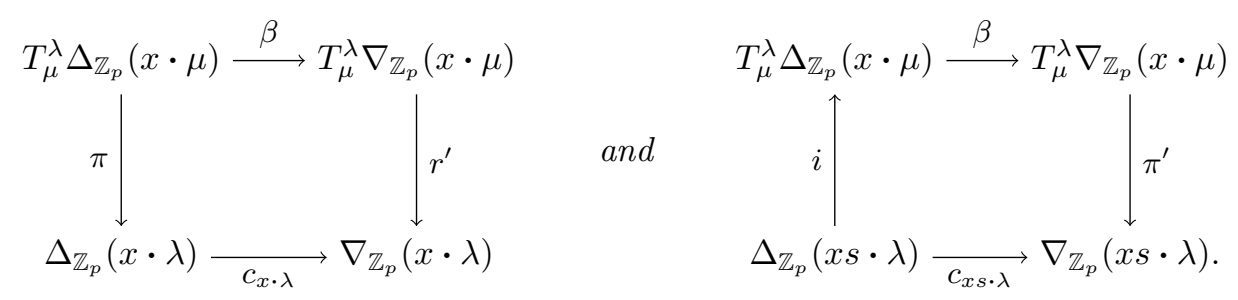

Proof. The short exact sequence

$$
0 \longrightarrow \nabla_{\mathbb{Z}_{p}}(x \cdot \lambda) \stackrel{i^{\prime}}{\longrightarrow} T_{\mu}^{\lambda} \nabla_{\mathbb{Z}_{p}}(x \cdot \mu) \stackrel{\pi^{\prime}}{\longrightarrow} \nabla_{\mathbb{Z}_{p}}(x s \cdot \lambda) \longrightarrow 0
$$

gives rise to an exact sequence

$$
\begin{aligned}
& 0 \rightarrow \operatorname{Hom}_{G_{\mathbb{Z}_{p}}}\left(\Delta_{\mathbb{Z}_{p}}(x \cdot \lambda), \nabla_{\mathbb{Z}_{p}}(x \cdot \lambda)\right) \\
& \rightarrow \operatorname{Hom}_{G_{\mathbb{Z}_{p}}}\left(\Delta_{\mathbb{Z}_{p}}(x \cdot \lambda), T_{\mu}^{\lambda} \nabla_{\mathbb{Z}_{p}}(x \cdot \mu)\right) \\
& \quad \rightarrow \operatorname{Hom}_{G_{\mathbb{Z}_{p}}}\left(\Delta_{\mathbb{Z}_{p}}(x \cdot \lambda), \nabla_{\mathbb{Z}_{p}}(x s \cdot \lambda)\right)=0
\end{aligned}
$$

so the map $\varphi \mapsto \operatorname{adj}_{1}\left(i^{\prime} \circ \varphi\right)$ gives an isomorphism

$$
\begin{aligned}
\operatorname{Hom}_{G_{\mathbb{Z}_{p}}}\left(\Delta_{\mathbb{Z}_{p}}(x \cdot \lambda), \nabla_{\mathbb{Z}_{p}}(x \cdot \lambda)\right) & \cong \operatorname{Hom}_{G_{\mathbb{Z}_{p}}}\left(\Delta_{\mathbb{Z}_{p}}(x \cdot \lambda), T_{\mu}^{\lambda} \nabla_{\mathbb{Z}_{p}}(x \cdot \mu)\right) \\
& \cong \operatorname{Hom}_{G_{\mathbb{Z}_{p}}}\left(T_{\lambda}^{\mu} \Delta_{\mathbb{Z}_{p}}(x \cdot \lambda), \nabla_{\mathbb{Z}_{p}}(x \cdot \mu)\right) \\
& \cong \operatorname{Hom}_{G_{\mathbb{Z}_{p}}}\left(\Delta_{\mathbb{Z}_{p}}(x \cdot \mu), \nabla_{\mathbb{Z}_{p}}(x \cdot \mu)\right) .
\end{aligned}
$$


Hence up to a unit in $\mathbb{Z}_{p}$, we have

$$
c_{x \cdot \mu}=\operatorname{adj}_{1}\left(i^{\prime} \circ c_{x \cdot \lambda}\right)=\operatorname{adj}_{1}\left(i^{\prime}\right) \circ T_{\lambda}^{\mu} c_{x \cdot \lambda}=\operatorname{id}_{\nabla_{\mathbb{Z}_{p}}(\mu)} \circ T_{\lambda}^{\mu} c_{x \cdot \lambda}=T_{\lambda}^{\mu} c_{x \cdot \lambda}
$$

matching the first claim. Commutativity of the diagrams follows from Lemma 2.5 in And97.

Remark 5.3. The first statement in Lemma $\$ 5.2$ is true in greater generality, as has been pointed out by the referee: For $\delta, \nu \in C_{\text {fund }}$ such that $x \cdot \nu \in X^{+}$and $x \cdot \nu$ belongs to the upper closure of the facet containing $x \cdot \delta$, we have $T_{\delta}^{\nu} c_{x \cdot \delta}=c_{x \cdot \nu}$. (See Section II.6.2 in Jan03] for the terminology.)

Indeed, by Proposition II.7.13 and Lemma II.B.9 in [Jan03, the $G_{\mathbb{Z}_{p}}$-module $T_{\nu}^{\delta} \nabla_{\mathbb{Z}_{p}}(x \cdot \nu)$ has a filtration with subquotients the induced modules $\nabla_{\mathbb{Z}_{p}}(x w \cdot \delta)$ for $w \in \operatorname{Stab}_{W_{\text {aff }}}(\nu)$, each weight $x w \cdot \delta$ occurring precisely once. As $x \cdot \delta$ is minimal among these weights, the first submodule in this filtration can be chosen to be isomorphic to $\nabla_{\mathbb{Z}_{p}}(x \cdot \delta)$ (see Remark 4 in Section II.4.16 in [Jan03]), so there is a short exact sequence

$$
0 \longrightarrow \nabla_{\mathbb{Z}_{p}}(x \cdot \delta) \longrightarrow T_{\nu}^{\delta} \nabla(x \cdot \delta) \longrightarrow M \longrightarrow 0 .
$$

Now the claim follows as in the proof of Lemma 5.2 .

5.2. Cokernels and exact sequences. Recall from Section 1 that every homomorphism of $\mathbb{Z}_{p^{-}}$modules $\varphi: M \rightarrow N$ gives rise to a filtration

$$
M=F^{0}(\varphi) \supseteq F^{1}(\varphi) \supseteq F^{2}(\varphi) \supseteq \cdots
$$

with $F^{i}(\varphi):=\varphi^{-1}\left(p^{i} \cdot N\right)$ for $i \geq 0$. We write $\bar{F}^{i}(\varphi)$ for the subspace spanned by the image of $F^{i}(\varphi)$ in $M \otimes \mathbb{F}$. Under some additional hypotheses on $\varphi$, we can give an alternative description of the length of this filtration and of the sum of the dimensions of the vector spaces $\bar{F}^{i}(\varphi)$ for $i>0$.

Definition 5.4. Let $M$ be a finitely generated torsion $\mathbb{Z}_{p}$-module. Then $M \cong$ $\prod_{i=1}^{r} \mathbb{Z}_{p} / p^{d_{i}} \mathbb{Z}_{p}$ for certain positive integers $d_{1}, \ldots, d_{r}$ and we write

$$
\ell(M)=\sum_{i=1}^{r} d_{i} \quad \text { and } \quad \operatorname{tmax}(M)=\max \left\{d_{i} \mid 1 \leq i \leq r\right\}
$$

for the composition length and the maximal torsion of $M$, respectively.

Let us write $\mathbb{Q}_{p}$ for the field of $p$-adic numbers. Lemma [5.5] is well-known, see for instance Section II.8.18 in [Jan03].

Lemma 5.5. Let $M$ and $N$ be free $\mathbb{Z}_{p}$-modules of finite rank and let $\varphi: M \rightarrow N$ be a homomorphism of $\mathbb{Z}_{p}$-modules such that $\varphi \otimes \mathbb{Q}_{p}$ is an isomorphism. Then $\operatorname{cok}(\varphi)$ is a torsion $\mathbb{Z}_{p}$-module and

$$
\sum_{i>0} \operatorname{dim} \bar{F}^{i}(\varphi)=\ell(\operatorname{cok}(\varphi)) \quad \text { and } \quad \max \left\{i \mid \bar{F}^{i}(\varphi) \neq 0\right\}=\operatorname{tmax}(\operatorname{cok}(\varphi)) .
$$

Proof. By applying the exact functor $-\otimes \mathbb{Q}_{p}$ to the exact sequence

$$
0 \rightarrow \operatorname{ker}(\varphi) \rightarrow M \stackrel{\varphi}{\longrightarrow} N \rightarrow \operatorname{cok}(\varphi) \rightarrow 0
$$

and using the fact that a $\mathbb{Z}_{p}$-module $M^{\prime}$ is torsion if and only if $M^{\prime} \otimes \mathbb{Q}_{p} \cong 0$, we see that $\operatorname{ker}(\varphi)$ and $\operatorname{cok}(\varphi)$ are torsion $\mathbb{Z}_{p}$-modules. As $M$ is free, it follows that $\varphi$ is injective and by the elementary divisor theorem, we can choose $\mathbb{Z}_{p}$-bases 
$m_{1}, \ldots, m_{r}$ of $M$ and $n_{1}, \ldots, n_{r}$ of $N$ such that $\varphi\left(m_{i}\right)=a_{i} \cdot n_{i}$ for some $0 \neq a_{i} \in \mathbb{Z}_{p}$ for all $i$. Then $F^{j}(\varphi)$ has a basis given by

$$
\left\{m_{i} \mid \nu_{p}\left(a_{i}\right) \geq j\right\} \cup\left\{p^{j-\nu_{p}\left(a_{i}\right)} \cdot m_{i} \mid \nu_{p}\left(a_{i}\right)<j\right\},
$$

so $\left\{m_{i} \otimes 1 \mid \nu_{p}\left(a_{i}\right) \geq j\right\}$ is a basis of the vector space $\bar{F}^{j}(\varphi)$ and it follows that

$$
\begin{aligned}
\sum_{j>0} \operatorname{dim}\left(\bar{F}^{j}(\varphi)\right) & =\sum_{j>0}\left|\left\{i \mid \nu_{p}\left(a_{i}\right) \geq j\right\}\right| \\
& =\left|\left\{(i, j) \mid \nu_{p}\left(a_{i}\right) \geq j\right\}\right| \\
& =\sum_{i=1}^{r} \nu_{p}\left(a_{i}\right) \\
& =\ell(\operatorname{cok}(\varphi)) .
\end{aligned}
$$

Furthermore, the description of the bases of the vector spaces $\bar{F}^{j}(\varphi)$ implies that

$$
\max \left\{j \geq 0 \mid \bar{F}^{j}(\varphi) \neq 0\right\}=\max \left\{\nu_{p}\left(a_{i}\right) \mid 1 \leq i \leq r\right\}=\operatorname{tmax}(\operatorname{cok}(\varphi)),
$$

as claimed.

Every rational $G_{\mathbb{Z}_{p}}$-module $M$ has a weight space decomposition $M=\bigoplus_{\delta \in X} M_{\delta}$. If $M$ is a torsion module then so are all of the weight spaces and this allows us to define a character for $M$.

Definition 5.6. Let $M$ be a torsion $G_{\mathbb{Z}_{p}}$-module. The torsion character of $M$ is

$$
\operatorname{tch} M:=\sum_{\delta \in X} \ell\left(M_{\delta}\right) \cdot e^{\delta} \in \mathbb{Z}[X]^{W_{\text {fin }}} .
$$

Remark 5.7. Let us write $\operatorname{Rep}^{\text {tor }}\left(G_{\mathbb{Z}_{p}}\right)$ for the category of finitely generated torsion $G_{\mathbb{Z}_{p}}$-modules and $\left[\operatorname{Rep}^{\text {tor }}\left(G_{\mathbb{Z}_{p}}\right)\right]$ for its Grothendieck group. It is straightforward to see from the definition that characters of torsion $G_{\mathbb{Z}_{p}}$-modules are additive on short exact sequences, so the torsion character defines an isomorphism between $\left[\operatorname{Rep}^{\text {tor }}\left(G_{\mathbb{Z}_{p}}\right)\right]$ and $\mathbb{Z}[X]^{W_{\text {fin }}}$ that sends the isomorphism class of the torsion $G_{\mathbb{Z}_{p}}$ module $\nabla_{\mathbb{Z}_{p}}(\lambda) \otimes\left(\mathbb{Z}_{p} / p \mathbb{Z}_{p}\right)$ to $\chi_{\lambda}$ for all $\lambda \in X^{+}$. (Indeed, all irreducible $G_{\mathbb{Z}_{p}}{ }^{-}$ modules are torsion modules over $\mathbb{Z}_{p}$ and remain irreducible under extension of scalars to $\mathbb{F}$, so the torsion character takes a basis of $\left[\operatorname{Rep}^{\text {tor }}\left(G_{\mathbb{Z}_{p}}\right)\right]$ to a basis of $\mathbb{Z}[X]^{W_{\text {fin }}}$ because the corresponding statement is true for $[\operatorname{Rep}(G)]$.)

Lemma 5.8. Let $\varphi: M \rightarrow N$ be a homomorphism of $G_{\mathbb{Z}_{p}}$-modules. Further suppose that $M$ and $N$ are free of finite rank over $\mathbb{Z}_{p}$ and that $\varphi \otimes \mathbb{Q}_{p}$ is an isomorphism. Then

$$
\sum_{i>0} \operatorname{ch} \bar{F}^{i}(\varphi)=\operatorname{tch} \operatorname{cok}(\varphi)
$$

Proof. By Lemma 5.5, the $G_{\mathbb{Z}_{p}}-\operatorname{module} \operatorname{cok}(\varphi)$ is torsion, so that tch $\operatorname{cok}(\varphi)$ is welldefined. For $\delta \in X$, denote by $\varphi_{\delta}: M_{\delta} \rightarrow N_{\delta}$ the restriction of $\varphi$ to the $\delta$-weight spaces of $M$ and $N$. As $M$ and $N$ are free of finite rank, so are $M_{\delta}$ and $N_{\delta}$, and as $\varphi \otimes \mathbb{Q}_{p}$ is an isomorphism, so is $\varphi_{\delta} \otimes \mathbb{Q}_{p}$. The filtration $F^{\bullet}(\varphi)$ is compatible with the weight space decomposition (that is $F^{i}\left(\varphi_{\delta}\right)=F^{i}(\varphi)_{\delta}$ ) and Lemma 5.5 implies that

$$
\sum_{i>0} \operatorname{dim} \bar{F}^{i}(\varphi)_{\delta}=\sum_{i>0} \operatorname{dim} \bar{F}^{i}\left(\varphi_{\delta}\right)=\ell\left(\operatorname{cok}\left(\varphi_{\delta}\right)\right)=\ell\left(\operatorname{cok}(\varphi)_{\delta}\right) .
$$

Now the claim is immediate from the definition of torsion characters. 
Corollary 5.9. For $\delta \in X^{+}$, we have

$$
\mathrm{JSF}_{\delta}=\operatorname{tch}\left(\operatorname{cok}\left(c_{\delta}\right)\right) .
$$

Proof. By definition, we have $\operatorname{JSF}_{\delta}=\sum_{i>0} \operatorname{ch} \Delta(\delta)^{i}$, where $\Delta(\delta)^{i}=\bar{F}^{i}\left(c_{\delta}\right)$. Now $c_{\delta} \otimes \mathbb{Q}_{p}$ is an isomorphism between the simple $G_{\mathbb{Q}_{p}}$-modules $\Delta_{\mathbb{Q}_{p}}(\delta)$ and $\nabla_{\mathbb{Q}_{p}}(\delta)$ of highest weight $\delta$ and the claim follows from Lemma 5.8.

By Remark 5.7 and Corollary 5.9, we can compute $\mathrm{JSF}_{\delta}$ by computing the class $\left[\operatorname{cok}\left(c_{\delta}\right)\right]$ in the Grothendieck group of finitely generated torsion $G_{\mathbb{Z}_{p}}$-modules, for all $\delta \in X^{+}$.

As before, let us fix weights $\lambda \in C_{\text {fund }} \cap X$ and $\mu \in \bar{C}_{\text {fund }} \cap X$ such that $\operatorname{Stab}_{W_{\text {aff }}}(\mu)=\{1, s\}$. The $s$-wall crossing functor on $\operatorname{Rep}_{\lambda}(G)$ is defined as

$$
\Theta_{s}=T_{\mu}^{\lambda} \circ T_{\lambda}^{\mu}
$$

Writing $\operatorname{Rep}_{\lambda}^{\text {tor }}\left(G_{\mathbb{Z}_{p}}\right)$ for the category of finitely generated torsion $G_{\mathbb{Z}_{p}}$-modules in $\operatorname{Rep}_{\lambda}\left(G_{\mathbb{Z}_{p}}\right)$, there is an isomorphism of abelian groups

$$
\left[\operatorname{Rep}_{\lambda}^{\text {tor }}\left(G_{\mathbb{Z}_{p}}\right)\right] \longrightarrow M_{\text {asph }}
$$

with $\left[\nabla_{\mathbb{Z}_{p}}(x \cdot \lambda) \otimes\left(\mathbb{Z}_{p} / p \mathbb{Z}_{p}\right)\right] \mapsto N_{x}$. The latter can be upgraded to an isomorphism of $\mathbb{Z}\left[W_{\text {aff }}\right]$-modules by setting $[M] \cdot(s+1):=\left[\Theta_{s} M\right]$ for $s \in S$ and for a $G_{\mathbb{Z}_{p}}$-module $M$ in $\operatorname{Rep}_{\lambda}^{\text {tor }}\left(G_{\mathbb{Z}_{p}}\right)$. For the remainder of this section, we write $\Delta_{x}=\Delta_{\mathbb{Z}_{p}}(x \cdot \lambda)$, $\nabla_{x}=\nabla_{\mathbb{Z}_{p}}(x \cdot \lambda)$ and $c_{x}=c_{x \cdot \lambda}: \Delta_{x} \rightarrow \nabla_{x}$ for all $x \in W_{\text {aff }}^{+}$. For $m>0$, we set $\nabla_{x} / p^{m}:=\nabla_{x} \otimes\left(\mathbb{Z}_{p} / p^{m} \mathbb{Z}_{p}\right)$ so that $\left[\nabla_{x} / p^{m}\right]=m \cdot\left[\nabla_{x} / p\right]$.

Theorem 5.10 gives a representation theoretic explanation for the (combinatorial) recursion formula from Theorem 4.1

Theorem 5.10. Let $x \in W_{\mathrm{aff}}^{+}$and $s \in S$ such that $x<x s \in W_{\mathrm{aff}}^{+}$. Then there exist torsion $G_{\mathbb{Z}_{p}}$-modules $A, B, C, D$ and $E$ and exact sequences

$$
\begin{aligned}
0 & \longrightarrow A \longrightarrow \Theta_{s} \operatorname{cok}\left(c_{x}\right) \longrightarrow C \longrightarrow 0, \\
0 \longrightarrow B & \longrightarrow \operatorname{cok}\left(c_{x s}\right) \longrightarrow C \longrightarrow 0, \\
0 \longrightarrow D \longrightarrow & \longrightarrow \operatorname{cok}\left(c_{x}\right) \longrightarrow E \longrightarrow 0, \\
0 \longrightarrow D \longrightarrow & \longrightarrow \nabla_{x} / p^{m} \longrightarrow E \longrightarrow 0,
\end{aligned}
$$

where $m=\nu_{p}\left(m\left(x s x^{-1}\right) \cdot p\right)$. In particular, we have

$$
\left[\operatorname{cok}\left(c_{x s}\right)\right]=\left[\Theta_{s} \operatorname{cok}\left(c_{x}\right)\right]-\left[\operatorname{cok}\left(c_{x}\right)\right]+\left[\nabla_{x} / p^{m}\right]=\left[\operatorname{cok}\left(c_{x}\right)\right] \cdot s+m \cdot\left[\nabla_{x} / p\right]
$$

in the Grothendieck group of torsion $G_{\mathbb{Z}_{p}}$-modules.

Proof. Recall that we have fixed $\mu \in \bar{C}_{\text {fund }} \cap X$ with $\operatorname{Stab}_{W_{\text {aff }}}(\mu)=\{1, s\}$ and note that the unique reflection hyperplane containing $x \cdot \mu$ is the one corresponding to $x s x^{-1}$. Using the Weyl dimension formula (and the fact that $p \geq h$ ), it is straightforward to see that

$$
m=\nu_{p}\left(m\left(x s x^{-1}\right) \cdot p\right)=\nu_{p}(\operatorname{dim} \Delta(x \cdot \mu)) .
$$

We adopt notation from Lemmas 5.1 and 5.2, in particular $\beta=T_{\mu}^{\lambda} c_{x \cdot \mu}=\Theta_{s} c_{x}$ and

$$
\begin{gathered}
i=\operatorname{adj}_{1}^{-1}\left(\operatorname{id}_{\Delta_{\mathbb{Z}_{p}}(x \cdot \mu)}\right): \Delta_{x s} \longrightarrow \Theta_{s} \Delta_{x}, \\
r^{\prime}=\operatorname{adj}_{2}\left(\operatorname{id}_{\nabla_{\mathbb{Z}_{p}}(x \cdot \mu)}\right): \Theta_{s} \nabla_{x} \longrightarrow \nabla_{x}, \\
\pi^{\prime}=\operatorname{adj}_{2}\left(\operatorname{id}_{\nabla_{\mathbb{Z}_{p}}(x \cdot \mu)}\right): \Theta_{s} \nabla_{x} \longrightarrow \nabla_{x s} .
\end{gathered}
$$


Furthermore, we denote by $q: \Theta_{s} \Delta_{x} \rightarrow \Theta_{s} \Delta_{x} / \operatorname{ker}\left(\pi^{\prime} \circ \beta\right)$ the canonical quotient homomorphism and by $\vartheta: \Theta_{s} \Delta_{x} / \operatorname{ker}\left(\pi^{\prime} \circ \beta\right) \rightarrow \nabla_{x s}$ the unique homomorphism such that $\pi^{\prime} \circ \beta=\vartheta \circ q$. Then there is a commutative diagram

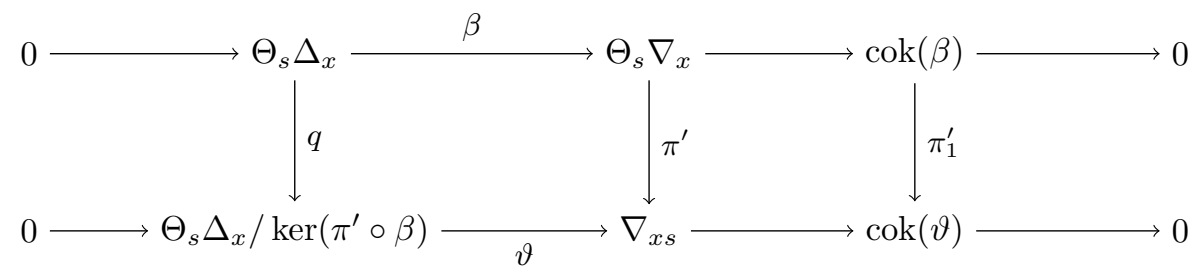

with exact rows, where $\pi_{1}^{\prime}$ is the homomorphism induced by $\pi^{\prime}$. As $q$ and $\pi^{\prime}$ are surjective, the snake lemma gives rise to an exact sequence

$$
0 \rightarrow \operatorname{ker}\left(\pi^{\prime} \circ \beta\right) \rightarrow \operatorname{ker}\left(\pi^{\prime}\right) \rightarrow \operatorname{ker}\left(\pi_{1}^{\prime}\right) \rightarrow 0 \rightarrow 0 \rightarrow \operatorname{cok}\left(\overline{\pi^{\prime}}\right) \rightarrow 0,
$$

in particular $\operatorname{cok}\left(\pi_{1}^{\prime}\right)=0$ and we obtain a short exact sequence

$$
0 \rightarrow \operatorname{ker}\left(\pi_{1}^{\prime}\right) \rightarrow \operatorname{cok}(\beta) \rightarrow \operatorname{cok}(\vartheta) \rightarrow 0 .
$$

By exactness of $\Theta_{s}$, we have $\operatorname{cok}(\beta)=\operatorname{cok}\left(\Theta_{s} c_{x}\right) \cong \Theta_{s} \operatorname{cok}\left(c_{x}\right)$ and with $A:=$ $\operatorname{ker}\left(\pi_{1}^{\prime}\right)$ and $C:=\operatorname{cok}(\vartheta)$, we obtain the first exact sequence (5.1). By the second commutative diagram in Lemma 5.2, we have another commutative diagram

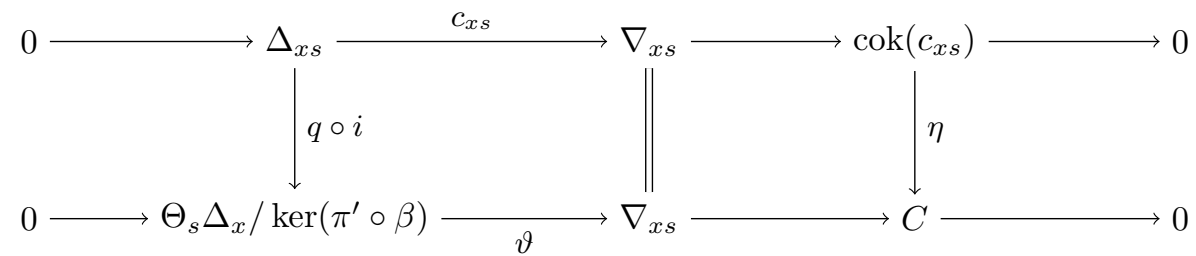

with exact rows, where $\eta$ is the induced homomorphism on the cokernels. This affords an exact sequence

$$
0 \rightarrow \operatorname{ker}(\eta) \rightarrow \operatorname{cok}(q \circ i) \rightarrow 0 \rightarrow \operatorname{cok}(\eta) \rightarrow 0,
$$

so $\operatorname{cok}(\eta)=0$ and $\operatorname{ker}(\eta) \cong \operatorname{cok}(q \circ i)$. With $B:=\operatorname{ker}(\eta) \cong \operatorname{cok}(q \circ i)$, we obtain our second exact sequence

$$
0 \rightarrow B \rightarrow \operatorname{cok}\left(c_{x s}\right) \rightarrow C \rightarrow 0 .
$$

Next note that we have a commutative diagram

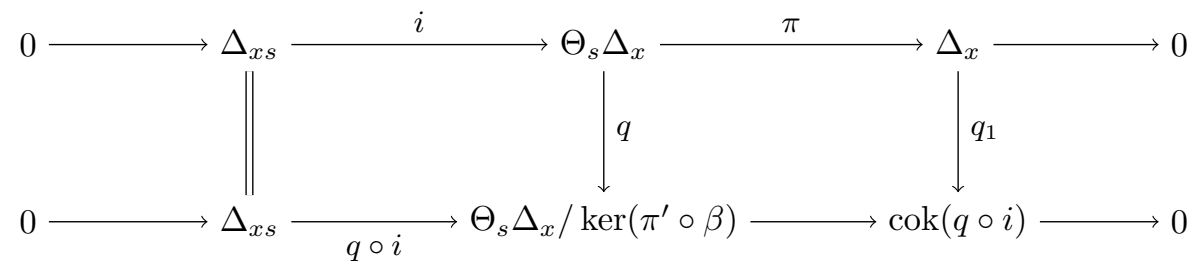

with exact rows, where $q_{1}$ is induced by $q$. Therefore we have an exact sequence

$$
0 \rightarrow \operatorname{ker}\left(\pi^{\prime} \circ \beta\right) \rightarrow \operatorname{ker}\left(q_{1}\right) \rightarrow 0 \rightarrow 0 \rightarrow \operatorname{cok}\left(q_{1}\right) \rightarrow 0,
$$


so $\operatorname{cok}\left(q_{1}\right)=0$ and $\operatorname{ker}\left(\pi^{\prime} \circ \beta\right) \cong \operatorname{ker}\left(q_{1}\right)$. Writing $\pi_{0}: \operatorname{ker}\left(\pi^{\prime} \circ \beta\right) \rightarrow \Delta_{\mathbb{Z}_{p}}(x \cdot \lambda)$ for the restriction of $\pi$ to $\operatorname{ker}\left(\pi^{\prime} \circ \beta\right)$, we further have a short exact sequence

$$
0 \rightarrow \operatorname{ker}\left(\pi_{0}\right) \stackrel{\pi_{0}}{\longrightarrow} \Delta_{\mathbb{Z}_{p}}(x s \cdot \lambda) \rightarrow \operatorname{cok}(q \circ i) \rightarrow 0
$$

and it follows that $B \cong \operatorname{cok}(q \circ i) \cong \operatorname{cok}\left(\pi_{0}\right)$. Next, denote by

$$
\beta_{0}: \operatorname{ker}\left(\pi^{\prime} \circ \beta\right) \rightarrow \operatorname{ker}\left(\pi^{\prime}\right)
$$

the restriction of $\beta$ to $\operatorname{ker}\left(\pi^{\prime} \circ \beta\right)$ and note that the short exact sequence

$$
0 \rightarrow \operatorname{ker}\left(\pi^{\prime} \circ \beta\right) \stackrel{\beta_{0}}{\longrightarrow} \operatorname{ker}\left(\pi^{\prime}\right) \rightarrow \operatorname{ker}\left(\pi_{1}^{\prime}\right) \rightarrow 0
$$

from (5.5) yields an isomorphism $A=\operatorname{ker}\left(\pi_{1}^{\prime}\right) \cong \operatorname{cok}\left(\beta_{0}\right)$. Finally, let

$$
r_{0}^{\prime}: \operatorname{ker}\left(\pi^{\prime}\right) \rightarrow \nabla_{x}
$$

be the restriction of $r^{\prime}$ to $\operatorname{ker}\left(\pi^{\prime}\right)$. Using the first commutative diagram in Lemma 5.2, we obtain a commutative diagram

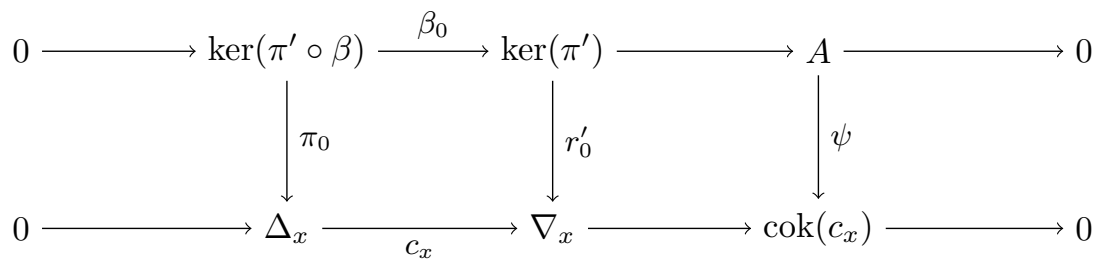

with exact rows, where $\psi$ is induced by $r_{0}^{\prime}$. Now we have $\operatorname{ker}\left(\pi^{\prime}\right)=i^{\prime}\left(\nabla_{x s}\right)$ and $r^{\prime} \circ i^{\prime}=p^{m} \cdot \operatorname{id}_{\nabla_{x}}$ by Lemma 5.1, so $r_{0}^{\prime}$ is injective with

$$
\operatorname{cok}\left(r_{0}^{\prime}\right) \cong \operatorname{cok}\left(r^{\prime} \circ i^{\prime}\right) \cong \nabla_{x} / p^{m} .
$$

As $B \cong \operatorname{cok}\left(\pi_{0}\right)$, the above diagram affords exact sequences

$$
0 \rightarrow \operatorname{ker}(\psi) \rightarrow B \rightarrow \nabla_{x} / p^{m} \rightarrow \operatorname{cok}(\psi) \rightarrow 0
$$

and

$$
0 \rightarrow \operatorname{ker}(\psi) \rightarrow A \rightarrow \operatorname{cok}\left(c_{x}\right) \rightarrow \operatorname{cok}(\psi) \rightarrow 0
$$

and with $D:=\operatorname{ker}(\psi)$ and $E:=\operatorname{cok}(\psi)$, we obtain the exact sequences (5.3) and (5.4). The final claim easily follows from (5.1)-(5.4) as

$$
\begin{aligned}
{\left[\operatorname{cok}\left(c_{x s}\right)\right] } & =[B]+[C] \\
& =[B]+\left[\Theta_{s} \operatorname{cok}\left(c_{x}\right)\right]-[A] \\
& =[B]+\left[\Theta_{s} \operatorname{cok}\left(c_{x}\right)\right]-\left[\operatorname{cok}\left(c_{x}\right)\right]-[D]+[E] \\
& =\left[\Theta_{s} \operatorname{cok}\left(c_{x}\right)\right]-\left[\operatorname{cok}\left(c_{x}\right)\right]+\left[\nabla_{x} / p^{m}\right],
\end{aligned}
$$

where $\left[\nabla_{x} / p^{m}\right]=m \cdot\left[\nabla_{x} / p\right]$ and $\left[\Theta_{s} \operatorname{cok}\left(c_{x}\right)\right]=\left[\operatorname{cok}\left(c_{x}\right)\right] \cdot(s+1)$.

Corollary 5.11. Let $\lambda \in C_{\text {fund }} \cap X, x \in W_{\text {aff }}^{+}$and $s \in S$ such that $x<x s \in W_{\text {aff }}^{+}$. Then

$$
\max \left\{i \geq 0 \mid \Delta(x s \cdot \lambda)^{i} \neq 0\right\} \leq 2 \cdot \max \left\{i \geq 0 \mid \Delta(x \cdot \lambda)^{i} \neq 0\right\}+m,
$$

where $m=\nu_{p}\left(m\left(x s x^{-1}\right) \cdot p\right)$. 
Proof. Recall from Lemma 5.5 that

$$
\max \left\{i \geq 0 \mid \Delta(x s \cdot \lambda)^{i} \neq 0\right\}=\operatorname{tmax}\left(\operatorname{cok}\left(c_{x s}\right)\right)
$$

and

$$
\max \left\{i \geq 0 \mid \Delta(x \cdot \lambda)^{i} \neq 0\right\}=\operatorname{tmax}\left(\operatorname{cok}\left(c_{x}\right)\right) .
$$

For a short exact sequence of torsion $\mathbb{Z}_{p}$-modules $0 \rightarrow M^{\prime} \rightarrow M \rightarrow M^{\prime \prime} \rightarrow 0$, we have

$$
\max \left\{\operatorname{tmax}\left(M^{\prime}\right), \operatorname{tmax}\left(M^{\prime \prime}\right)\right\} \leq \operatorname{tmax}(M) \leq \operatorname{tmax}\left(M^{\prime}\right)+\operatorname{tmax}\left(M^{\prime \prime}\right),
$$

so (5.1)-(5.4) imply that

$$
\begin{array}{r}
\operatorname{tmax}\left(\operatorname{cok}\left(c_{x s}\right)\right) \leq \operatorname{tmax}(B)+\operatorname{tmax}(C) \leq \operatorname{tmax}(D)+m+\operatorname{tmax}(C) \\
\leq \operatorname{tmax}(A)+m+\operatorname{tmax}(C) \leq 2 \cdot \operatorname{tmax}\left(\Theta_{s} \operatorname{cok}\left(c_{x}\right)\right)+m .
\end{array}
$$

As $\Theta_{s} \operatorname{cok}\left(c_{x}\right)$ is a direct summand of a tensor product of $\operatorname{cok}\left(c_{x}\right)$ with some other $G_{\mathbb{Z}_{p}}$-module, we have $\operatorname{tmax}\left(\Theta_{s} \operatorname{cok}\left(c_{x}\right)\right) \leq \operatorname{tmax}\left(\operatorname{cok}\left(c_{x}\right)\right)$ and it follows that

$$
\operatorname{tmax}\left(\operatorname{cok}\left(c_{x s}\right)\right) \leq 2 \cdot \operatorname{tmax}\left(\operatorname{cok}\left(c_{x}\right)\right)+m,
$$

as required.

Remark 5.12. If one assumes the Jantzen conjecture (stated in Section II.C.9 of Jan03 as the condition $(F, w, s)^{+}$) then a result of $\mathrm{H}$. H. Andersen shows that the composition multiplicities in the layers of the Jantzen filtration are given by coefficients of inverse parabolic Kazhdan-Lusztig polynomials, see Theorem 2.4 in And87. In this case, it turns out that the factor 2 in the upper bound in Corollary 5.11 is superfluous. Note that the Jantzen conjecture would imply the Lusztig character formula, so this can only be expected to happen in the so-called Jantzen region and when $p$ is very large.

Remark 5.13. The following alternative way of obtaining an upper bound on the length of the Jantzen filtration has been pointed out to the author by $\mathrm{H}$. H. Andersen: For $\nu \in X^{+}$, one can mimic the construction of the homomorphism $c_{\nu}: \Delta_{\mathbb{Z}_{p}}(\nu) \rightarrow \nabla_{\mathbb{Z}_{p}}(\nu)$ from Section II.8 in Jan03 to define a homomorphism

$$
c_{\nu}^{\prime}: \nabla_{\mathbb{Z}_{p}}(\lambda) \rightarrow \Delta_{\mathbb{Z}_{p}}(\lambda)
$$

such that

$$
c_{\nu} \circ c_{\nu}^{\prime}=\left(\prod_{\beta \in \Phi^{+}}\left(\left\langle\nu+\rho, \beta^{\vee}\right\rangle-1\right) !\right) \cdot \operatorname{id}_{\nabla_{\mathbb{Z}_{p}}(\nu)} .
$$

Note that J. C. Jantzen works over the integers rather than the $p$-adic integers. The homomorphism corresponding to $c_{\nu}$ is called $\tilde{T}_{w_{0}}\left(w_{0} \cdot \nu\right)$ and defined in Section II.8.16 in Jan03. In order to define the homomorphism $c_{\nu}^{\prime}$, one replaces the homomorphism from II.8.15(3) in Jan03 by the homomorphism from II.8.15(4); the identity for the composition of $c_{\nu}$ with $c_{\nu}^{\prime}$ follows from the remark after II.8.15(4). Now there is a canonical surjective homomorphism from $\operatorname{cok}\left(c_{\nu} \circ c_{\nu}^{\prime}\right)$ to $\operatorname{cok}\left(c_{\nu}\right)$ and it follows that

$$
\begin{aligned}
\max \left\{i \mid \Delta(\nu)^{i} \neq 0\right\} & =\operatorname{tmax}\left(\operatorname{cok}\left(c_{\nu}\right)\right) \leq \operatorname{tmax}\left(\operatorname{cok}\left(c_{\nu} \circ c_{\nu}^{\prime}\right)\right) \\
& =\nu_{p}\left(\prod_{\beta \in \Phi^{+}}\left(\left\langle\nu+\rho, \beta^{\vee}\right\rangle-1\right) !\right)=\sum_{\beta \in \Phi^{+}} \sum_{i=1}^{\left\langle\nu+\rho, \beta^{\vee}\right\rangle-1} \nu_{p}(i) .
\end{aligned}
$$


If $\nu$ is $p$-regular, say $\nu \in x \cdot C_{\text {fund }}$ for some $x \in W_{\text {aff }}^{+}$, then arguing as in the second paragraph of Section 3 , we get

$$
\max \left\{i \mid \Delta(\nu)^{i} \neq 0\right\} \leq \sum_{\beta \in \Phi^{+}} \sum_{i=1}^{\left\langle\nu+\rho, \beta^{\vee}\right\rangle} \nu_{p}(i)=\sum_{s \in R_{L}(x)} \nu_{p}(m(s) \cdot p) .
$$

If we further assume that $\left\langle\nu+\rho, \alpha_{\mathrm{h}}^{\vee}\right\rangle<p^{2}$ (so that $x \cdot C_{\text {fund }}$ lies in the lowest $p^{2}$-alcove) then $m(s)<p$ for all $s \in R_{L}(x)$ and it follows that

$$
\max \left\{i \mid \Delta(\nu)^{i} \neq 0\right\} \leq\left|R_{L}(x)\right|=\ell(x) .
$$

Neither of the bounds on the length of the Jantzen filtration from Corollary 5.11 and Remark 5.13 is sharp, except in special cases. Note that Corollary [5.11] can also be used to obtain an explicit bound on the length of the Jantzen filtration of a Weyl module $\Delta(\nu)$ with $\nu \in x \cdot C_{\text {fund }}$ for some $x \in W_{\text {aff }}^{+}$in terms of $\ell(x)$, but this bound is much bigger than the one that was given in Remark 5.13. By induction on $\ell(x)$, one obtains

$$
\max \left\{i \mid \Delta(\nu)^{i} \neq 0\right\} \leq 2^{\ell(x)}-1
$$

when $x \cdot C_{\text {fund }}$ lies in the lowest $p^{2}$-alcove. Corollary 5.11 becomes useful in situations where the length of the Jantzen filtration of a Weyl module (with $p$-regular highest weight) is already known and one wants to bound the length of the Jantzen filtration of a Weyl module with highest weight in an adjacent alcove.

5.3. Euler characteristics. For finitely generated $G_{\mathbb{Z}_{p}}$-modules $M$ and $N$, the $\mathbb{Z}_{p}$-module $\operatorname{Hom}_{G_{\mathbb{Z}_{p}}}(M, N)$ decomposes naturally as the direct sum of its torsion submodule $\operatorname{Hom}_{G_{\mathbb{Z}_{p}}}(M, N)_{\text {tor }}$ and its unique maximal (torsion-)free submodule $\operatorname{Hom}_{G_{\mathbb{Z}_{p}}}(M, N)_{\text {fr }}$. For all $i>0$, the Ext-group $\operatorname{Ext}_{G_{\mathbb{Z}_{p}}}^{i}(M, N)$ is a torsion $\mathbb{Z}_{p^{-}}$ module and following U. Kulkarni, we define the torsion Euler characteristic by

$$
E(M, N)=\ell\left(\operatorname{Hom}_{G_{\mathbb{Z}_{p}}}(M, N)_{\mathrm{tor}}\right)+\sum_{i>0}(-1)^{i} \cdot \ell\left(\operatorname{Ext}_{G_{\mathbb{Z}_{p}}}(M, N)\right) .
$$

Then U. Kulkarni shows in Section 1.4 of [Kul06] that

$$
\mathrm{JSF}_{\mu}=-\sum_{\lambda \in X^{+}} E\left(\Delta_{\mathbb{Z}_{p}}(\lambda), \Delta_{\mathbb{Z}_{p}}(\mu)\right) \cdot \chi_{\lambda}
$$

for all $\mu \in X^{+}$, which amounts to Kulkarni's formula

$$
\left\langle\chi_{\lambda}^{*}, \mathrm{JSF}_{\mu}\right\rangle=-E\left(\Delta_{\mathbb{Z}_{p}}(\lambda), \Delta_{\mathbb{Z}_{p}}(\mu)\right) .
$$

For a tilting module $T$ with good filtration multiplicities $a_{\mu}:=\operatorname{dim} \operatorname{Hom}_{G}(\Delta(\mu), T)$ for $\mu \in X^{+}$, it is shown in the proof of Theorem 5.2 in AK08 that

$$
\left\langle\mathrm{ASF}_{\lambda}, \operatorname{ch} T\right\rangle=-\sum_{\mu \in X^{+}} a_{\mu} \cdot E\left(\Delta_{\mathbb{Z}_{p}}(\lambda), \Delta_{\mathbb{Z}_{p}}(\mu)\right),
$$

so $\left\langle\mathrm{ASF}_{\lambda}, \chi_{\mu}\right\rangle=-E\left(\Delta_{\mathbb{Z}_{p}}(\lambda), \Delta_{\mathbb{Z}_{p}}(\mu)\right)=\left\langle\chi_{\lambda}^{*}, \mathrm{JSF}_{\mu}\right\rangle$. (This is the representation theoretic explanation of the duality formula mentioned in Remark 4.3.) Using the torsion Euler-characteristic, we can give yet another explanation for the recursion formula

$$
\mathrm{JSF}_{x s}=\nu_{p}\left(m\left(x s x^{-1}\right) \cdot p\right) \cdot N_{x}+\mathrm{JSF}_{x} \cdot s,
$$

via the following lemma. (We return to the notational conventions set up before Theorem 5.10, ) 
Lemma 5.14. Let $x, y \in W_{\mathrm{aff}}^{+}$and $s \in S$ such that $x<x s \in W_{\mathrm{aff}}^{+}$. Then

$$
E\left(\Delta_{y}, \Delta_{x s}\right)=E\left(\Delta_{y}, \Theta_{s} \Delta_{x}\right)-E\left(\Delta_{y}, \Delta_{x}\right)-\delta_{x, y} \cdot \nu_{p}\left(m\left(x s x^{-1}\right) \cdot p\right)
$$

and

$$
E\left(\Delta_{y}, \Theta_{s} \Delta_{x}\right)=E\left(\Theta_{s} \Delta_{y}, \Delta_{x}\right)= \begin{cases}E\left(\Delta_{y}, \Delta_{x}\right)+E\left(\Delta_{y s}, \Delta_{x}\right) & \text { if } y s \in W_{\mathrm{aff}}^{+}, \\ 0 & \text { otherwise }\end{cases}
$$

Proof. First, the short exact sequence

$$
0 \longrightarrow \Delta_{x s} \longrightarrow \Theta_{s} \Delta_{x} \longrightarrow \Delta_{x} \longrightarrow 0
$$

gives rise to a long exact sequence

$$
\begin{aligned}
0 \rightarrow & \operatorname{Hom}_{G_{\mathbb{Z}_{p}}}\left(\Delta_{y}, \Delta_{x s}\right) \rightarrow \operatorname{Hom}_{G_{\mathbb{Z}_{p}}}\left(\Delta_{y}, \Theta_{s} \Delta_{x}\right) \rightarrow \operatorname{Hom}_{G_{\mathbb{Z}_{p}}}\left(\Delta_{y}, \Delta_{x}\right) \\
& \rightarrow \operatorname{Ext}_{G_{\mathbb{Z}_{p}}}^{1}\left(\Delta_{y}, \Delta_{x s}\right) \rightarrow \operatorname{Ext}_{G_{\mathbb{Z}_{p}}}^{1}\left(\Delta_{y}, \Theta_{s} \Delta_{x}\right) \rightarrow \operatorname{Ext}_{G_{\mathbb{Z}_{p}}}^{1}\left(\Delta_{y}, \Delta_{x}\right) \rightarrow \cdots
\end{aligned}
$$

where the three initial terms are zero unless $y \in\{x, x s\}$ (see Section 1.2 in And97). If $y=x s$ then the third term in (5.7) is zero and the first term is isomorphic to the second, so the first claim follows for all $y \neq x$. For $y=x$, we have

$$
\operatorname{Ext}_{G_{\mathbb{Z}_{p}}}^{1}\left(\Delta_{x}, \Theta_{s} \Delta_{x}\right) \cong \operatorname{Ext}_{G_{\mathbb{Z}_{p}}}^{1}\left(T_{\lambda}^{\mu} \Delta_{x}, T_{\lambda}^{\mu} \Delta_{x}\right) \cong 0
$$

and the $\mathbb{Z}_{p}$-linear map from $\operatorname{Hom}_{G_{\mathbb{Z}_{p}}}\left(\Delta_{x}, \Theta_{s} \Delta_{x}\right) \cong \mathbb{Z}_{p}$ to $\operatorname{Hom}_{G_{\mathbb{Z}_{p}}}\left(\Delta_{x}, \Delta_{x}\right) \cong \mathbb{Z}_{p}$ identifies with multiplication by $p^{m}$ for $m=\nu_{p}\left(m\left(x s x^{-1}\right) \cdot p\right)$ by Lemma 5.1. Thus the beginning of the long exact sequence (5.7) reduces to

$$
0 \rightarrow 0 \rightarrow \mathbb{Z}_{p} \rightarrow \mathbb{Z}_{p} \rightarrow \mathbb{Z}_{p} / p^{m} \mathbb{Z}_{p} \rightarrow 0 \rightarrow \cdots
$$

and we conclude that

$$
E\left(\Delta_{x}, \Delta_{x s}\right)=E\left(\Delta_{x}, \Theta_{s} \Delta_{x}\right)-E\left(\Delta_{x}, \Delta_{x}\right)-m .
$$

The first equality in the second claim follows from the adjunction between $T_{\lambda}^{\mu}$ and $T_{\mu}^{\lambda}$. If $y s \notin W_{\text {aff }}^{+}$then $\Theta_{s} \Delta_{y}=0$ and therefore $E\left(\Theta_{s} \Delta_{y}, \Delta_{x}\right)=0$ as claimed, so now suppose that $y s \in W_{\text {aff }}^{+}$. In order to show the second equality, we may assume without loss of generality that $y<y s$ since $\Theta_{s} \Delta_{y} \cong \Theta_{s} \Delta_{y s}$. Then the short exact sequence

$$
0 \longrightarrow \Delta_{y s} \longrightarrow \Theta_{s} \Delta_{y} \longrightarrow \Delta_{y} \longrightarrow 0
$$

gives rise to a long exact sequence

$$
\begin{aligned}
0 \rightarrow & \operatorname{Hom}_{G_{\mathbb{Z}_{p}}}\left(\Delta_{y}, \Delta_{x}\right) \rightarrow \operatorname{Hom}_{G_{\mathbb{Z}_{p}}}\left(\Theta_{s} \Delta_{y}, \Delta_{x}\right) \rightarrow \operatorname{Hom}_{G_{\mathbb{Z}_{p}}}\left(\Delta_{y s}, \Delta_{x}\right) \\
& \rightarrow \operatorname{Ext}_{G_{\mathbb{Z}_{p}}}^{1}\left(\Delta_{y}, \Delta_{x}\right) \rightarrow \operatorname{Ext}_{G_{\mathbb{Z}_{p}}}^{1}\left(\Theta_{s} \Delta_{y}, \Delta_{x}\right) \rightarrow \operatorname{Ext}_{G_{\mathbb{Z}_{p}}}^{1}\left(\Delta_{y s}, \Delta_{x}\right) \rightarrow \cdots .
\end{aligned}
$$

Again, the three initial terms are zero unless $x \in\{y, y s\}$ and the condition $y<y s$ implies that $x \neq y s$. If $x=y$ then the third term in (5.8) is zero and the first term is isomorphic to the second, so the second claim follows in all cases.

Remark 5.15. Using Lemma 5.14 and Kulkarni's formula

$$
\left\langle N_{y}^{*}, \mathrm{JSF}_{x}\right\rangle=-E\left(\Delta_{y}, \Delta_{x}\right)
$$


from (5.6), we can recover the recursion formula as follows: Let $x, y \in W_{\text {aff }}^{+}$and $s \in S$ such that $x<x s \in W_{\text {aff }}^{+}$. If $y s \in W_{\text {aff }}^{+}$then

$$
\begin{aligned}
\left\langle N_{y}^{*}, \mathrm{JSF}_{x s}\right\rangle & =-E\left(\Delta_{y}, \Delta_{x s}\right) \\
& =-E\left(\Delta_{y}, \Theta_{s} \Delta_{x}\right)+E\left(\Delta_{y}, \Delta_{x}\right)+\delta_{x, y} \cdot \nu_{p}\left(m\left(x s x^{-1}\right) \cdot p\right) \\
& =-E\left(\Delta_{y s}, \Delta_{x}\right)+\delta_{x, y} \cdot \nu_{p}\left(m\left(x s x^{-1}\right) \cdot p\right) \\
& =\left\langle N_{y}^{*} \cdot s, \mathrm{JSF}_{x}\right\rangle+\left\langle N_{y}^{*}, \nu_{p}\left(m\left(x s x^{-1}\right) \cdot p\right) \cdot N_{x}\right\rangle \\
& =\left\langle N_{y}^{*}, \mathrm{JSF}_{x} \cdot s+\nu_{p}\left(m\left(x s x^{-1}\right) \cdot p\right) \cdot N_{x}\right\rangle
\end{aligned}
$$

and if $y s \notin W_{\text {aff }}^{+}$then

$$
\begin{aligned}
\left\langle N_{y}^{*}, \mathrm{JSF}_{x s}\right\rangle & =-E\left(\Delta_{y}, \Delta_{x s}\right) \\
& =-E\left(\Delta_{y}, \Theta_{s} \Delta_{x}\right)+E\left(\Delta_{y}, \Delta_{x}\right)+\delta_{x, y} \cdot \nu_{p}\left(m\left(x s x^{-1}\right) \cdot p\right) \\
& =E\left(\Delta_{y}, \Delta_{x}\right)+\delta_{x, y} \cdot \nu_{p}\left(m\left(x s x^{-1}\right) \cdot p\right) \\
& =\left\langle N_{y}^{*} \cdot s, \mathrm{JSF}_{x}\right\rangle+\left\langle N_{y}^{*}, \nu_{p}\left(m\left(x s x^{-1}\right) \cdot p\right) \cdot N_{x}\right\rangle \\
& =\left\langle N_{y}^{*}, \mathrm{JSF}_{x} \cdot s+\nu_{p}\left(m\left(x s x^{-1}\right) \cdot p\right) \cdot N_{x}\right\rangle
\end{aligned}
$$

since $N_{y}^{*} \cdot s=-N_{y}^{*}$. It follows that

$$
\mathrm{JSF}_{x s}=\mathrm{JSF}_{x} \cdot s+\nu_{p}\left(m\left(x s x^{-1}\right) \cdot p\right) \cdot N_{x},
$$

as claimed.

\section{An EXAMPLE}

Suppose that $G$ is of type $\mathrm{A}_{n}$ for some $n \geq 2$ and that $p \geq h=n+1$. Fix a labeling $\Delta=\left\{\alpha_{1}, \ldots, \alpha_{n}\right\}$ of the simple roots such that

$$
\left\langle\alpha_{i}, \alpha_{j}^{\vee}\right\rangle= \begin{cases}2 & \text { if } i=j \\ -1 & \text { if }|i-j|=1 \\ 0 & \text { otherwise }\end{cases}
$$

and write $S=\left\{s_{0}, s_{1}, \ldots, s_{n}\right\}$ with $s_{0}=s_{\alpha_{\mathrm{h}}, 1}$ and $s_{i}=s_{\alpha_{i}}$ for $i=1, \ldots, n$. A product of two simple reflections $s_{i}$ and $s_{j}$ with $i<j$ has order 3 if $j=i+1$ or $(i, j)=(0, n)$ and it has order 2 otherwise; see Section 4.7 in [Hum90]. We write $\omega_{1}, \ldots, \omega_{n} \in X^{+}$for the fundamental dominant weights with respect to $\Delta$, that is $\left\langle\omega_{i}, \alpha_{j}^{\vee}\right\rangle=\delta_{i j}$, and fix the weight $\lambda=(p-n-1) \cdot \omega_{n} \in C_{\text {fund }}$. We consider the elements $x_{0}:=e$ and $x_{i}:=s_{0} s_{1} \cdots s_{i-1} \in W_{\text {aff }}$ for $1 \leq i \leq n$. By induction on $i$, one sees that

$$
s_{1} \cdots s_{i-1} \cdot \lambda=\lambda-\alpha_{i-1}-2 \alpha_{i-2}-\cdots-(i-1) \alpha_{1}=\lambda+\omega_{i}-i \cdot \omega_{1}=: \lambda_{i}
$$

for $1 \leq i \leq n$. As $\alpha_{\mathrm{h}}=\alpha_{1}+\cdots+\alpha_{n}=\omega_{1}+\omega_{n}$ and $\alpha_{\mathrm{h}}^{\vee}=\alpha_{1}^{\vee}+\cdots+\alpha_{n}^{\vee}$, we further obtain

$$
\left\langle\lambda_{i}+\rho, \alpha_{\mathrm{h}}^{\vee}\right\rangle=\left\langle(p-n-1) \cdot \omega_{n}+\omega_{i}-i \cdot \omega_{1}+\rho, \alpha_{\mathrm{h}}^{\vee}\right\rangle=p-i
$$

and

$x_{i} \cdot \lambda=s_{0} \cdot \lambda_{i}=\lambda_{i}-\left(\left\langle\lambda_{i}+\rho, \alpha_{\mathrm{h}}^{\vee}\right\rangle-p\right) \cdot \alpha_{\mathrm{h}}=\lambda_{i}+i \cdot \alpha_{\mathrm{h}}=\omega_{i}+(p-n-1+i) \cdot \omega_{n}$ for $1 \leq i \leq n$. Note that $x_{i} \cdot \lambda-x_{i-1} \cdot \lambda=\alpha_{i}+\cdots+\alpha_{n}=$ : $\beta_{i}$ for $1 \leq i \leq n$. In fact, 
it is straightforward to see that $x_{i} \cdot \lambda=s_{\beta_{i}, 1} x_{i-1} \cdot \lambda$ and therefore

$$
x_{i-1} s_{i-1} x_{i-1}^{-1}=x_{i} x_{i-1}^{-1}=s_{\beta_{i}, 1} .
$$

Also note that $x_{n} \cdot \lambda=p \cdot \omega_{n}$ and therefore $x_{n} \cdot C_{\text {fund }}=C_{\text {fund }}+p \cdot \omega_{n}$.

We can now compute $\mathrm{JSF}_{x_{i}}$ for $0 \leq i \leq n$ using the recursion formula and induction on $i$.

Lemma 6.1. For $0 \leq i \leq n$, we have $\operatorname{JSF}_{x_{i}}=\sum_{j=1}^{i}(-1)^{j+1} \cdot N_{x_{i-j}}$.

Proof. For $i=0$, we have $x_{0}=e$ and $\Delta\left(x_{0} \cdot \mu\right)=\Delta(\mu)=L(\mu)$ for all $\mu \in C_{\text {fund }} \cap X$ by the linkage principle, so $\mathrm{JSF}_{x_{0}}=0$ as required. For $i=1$, the recursion formula from Theorem 4.1 yields

$$
\mathrm{JSF}_{x_{1}}=\mathrm{JSF}_{s_{0}}=N_{s_{0}}+\mathrm{JSF}_{e} \cdot s_{0}=N_{s_{0}} .
$$

Now suppose that $1<i \leq n$ and that the claim is true for $x_{i-1}$. We have

$$
x_{i}=x_{i-1} s_{i-1}>x_{i-1}
$$

and $\nu_{p}\left(m\left(x_{i-1} s_{i-1} x_{i-1}^{-1}\right) \cdot p\right)=1$ because $x_{i-1} s_{i-1} x_{i-1}^{-1}=s_{\beta_{i}, 1}$. By the induction hypothesis and the recursion formula, we have

$$
\mathrm{JSF}_{x_{i}}=N_{x_{i-1}}+\mathrm{JSF}_{x_{i-1}} \cdot s_{i-1}=N_{x_{i-1}}+\sum_{j=1}^{i-1}(-1)^{j+1} \cdot N_{x_{i-1-j}} \cdot s_{i-1} .
$$

Now $s_{i-1}$ commutes with $s_{k-1}$ for all $k<i-1$, hence

$$
N_{x_{k}} \cdot s_{i-1}=N_{x_{k} s_{i-1}}=N_{s_{i-1} x_{k}}=-N_{x_{k}}
$$

for $k<i-1$ and it follows that

$$
\begin{aligned}
\mathrm{JSF}_{x_{i}} & =N_{x_{i-1}}+\sum_{j=1}^{i-1}(-1)^{j+1} \cdot N_{x_{i-1-j}} \cdot s_{i-1} \\
& =N_{x_{i-1}}-\sum_{j=1}^{i-1}(-1)^{j+1} \cdot N_{x_{i-1-j}} \\
& =\sum_{j=1}^{i}(-1)^{j+1} \cdot N_{x_{i-j}},
\end{aligned}
$$

as claimed.

Recall from Section 3 that for every $\mu \in C_{\text {fund }} \cap X$, there is an isomorphism between $M_{\text {asph }}$ and the character lattice of $\operatorname{Rep}_{\mu}(G)$ that sends $N_{x}$ to $\chi(x \cdot \mu)$ and $\mathrm{JSF}_{x}$ to $\mathrm{JSF}_{x \cdot \mu}$ for all $x \in W_{\text {aff }}^{+}$. Thus the equation in Lemma 6.1 can be rewritten as

$\sum_{j>0} \operatorname{ch} \Delta\left(x_{i} \cdot \mu\right)^{j}=\operatorname{JSF}_{x_{i} \cdot \mu}=\sum_{j=1}^{i}(-1)^{j+1} \cdot \chi\left(x_{i-j} \cdot \mu\right)=\sum_{j=1}^{i}(-1)^{j+1} \cdot \operatorname{ch} \Delta\left(x_{i-j} \cdot \mu\right)$.

Using this observation, we can determine the composition series of the Weyl modules with highest weight in the alcoves $x_{i} \cdot C_{\text {fund }}$ for $1 \leq i \leq n$. 
Lemma 6.2. Let $\mu \in C_{\text {fund }} \cap X$ and $1 \leq i \leq n$. In the Grothendieck group of $\operatorname{Rep}(G)$, we have

$$
\left[\Delta\left(x_{i} \cdot \mu\right)\right]=\left[L\left(x_{i} \cdot \mu\right)\right]+\left[L\left(x_{i-1} \cdot \mu\right)\right] \quad \text { and } \quad\left[L\left(x_{i} \cdot \mu\right)\right]=\sum_{j=0}^{i}(-1)^{j} \cdot\left[\Delta\left(x_{i-j} \cdot \mu\right)\right] .
$$

Proof. We prove the claim by induction on $i$. For $i=1$, we have

$$
\sum_{j>0} \operatorname{ch} \Delta\left(x_{1} \cdot \mu\right)^{j}=\operatorname{JSF}_{x_{1} \cdot \mu}=\operatorname{ch} \Delta(\mu)=\operatorname{ch} L(\mu)
$$

because $\Delta(\mu)=L(\mu)$. This implies that $\Delta\left(x_{1} \cdot \mu\right)^{1} \cong L(\mu)$ and therefore

$$
\left[\Delta\left(x_{1} \cdot \mu\right)\right]=\left[L\left(x_{1} \cdot \mu\right)\right]+\left[L\left(x_{0} \cdot \mu\right)\right] \quad \text { and } \quad\left[L\left(x_{1} \cdot \mu\right)\right]=\left[\Delta\left(x_{1} \cdot \mu\right)\right]-\left[\Delta\left(x_{0} \cdot \mu\right)\right] \text {, }
$$

as claimed. Now suppose that $i>1$ and that

$$
\left[L\left(x_{i-1} \cdot \mu\right)\right]=\sum_{j=1}^{i}(-1)^{j+1} \cdot\left[\Delta\left(x_{i-j} \cdot \mu\right)\right] .
$$

Then we have

$$
\sum_{j>0} \operatorname{ch} \Delta\left(x_{i} \cdot \mu\right)^{j}=\operatorname{JSF}_{x_{i} \cdot \mu}=\sum_{j=1}^{i}(-1)^{j+1} \cdot \operatorname{ch} \Delta\left(x_{i-j} \cdot \mu\right)=\operatorname{ch} L\left(x_{i-1} \cdot \mu\right)
$$

and it follows that $\Delta\left(x_{i} \cdot \mu\right)^{1} \cong L\left(x_{i-1} \cdot \mu\right)$. As before, we conclude that

$$
\left[\Delta\left(x_{i} \cdot \mu\right)\right]=\left[L\left(x_{i} \cdot \mu\right)\right]+\left[L\left(x_{i-1} \cdot \mu\right)\right]
$$

and

$$
\left[L\left(x_{i} \cdot \mu\right)\right]=\left[\Delta\left(x_{i} \cdot \mu\right)\right]-\left[L\left(x_{i-1} \cdot \mu\right)\right]=\sum_{j=0}^{i}(-1)^{j} \cdot\left[\Delta\left(x_{i-j} \cdot \mu\right)\right],
$$

as required.

Remark 6.3. Lemmas 6.1 and 6.2 are actually special cases of a result of J. C. Jantzen, see the second paragraph of Section 7 in [Jan77]. For an element $x \in W_{\text {aff }}^{+}$ with reduced expression $x=t_{1} \cdots t_{m}$ and such that $x \cdot C_{\text {fund }}$ lies in the lowest $p^{2}$-alcove and the set $\left\{y \in W_{\text {aff }}^{+} \mid y<x\right\}$ is linearly ordered with respect to the Bruhat order, J. C. Jantzen shows (for all types of root systems) that the Jantzen sum formula is given by

$$
\mathrm{JSF}_{x}=\sum_{i=0}^{m-1}(-1)^{m-i-1} \cdot N_{t_{1} \cdots t_{i}} .
$$

As in Lemma 6.2, one concludes that a Weyl module with highest weight in the alcove $x \cdot C_{\text {fund }}$ has precisely two composition factors, with highest weights in $x \cdot C_{\text {fund }}$ and $t_{1} \cdots t_{m-1} \cdot C_{\text {fund }}$, respectively.

Let us conclude by illustrating the two upper bounds on the length of the Jantzen filtration from Corollary 5.11 and Remark 5.13 ,

Remark 6.4. From the proof of Lemma 6.2, we see that $\Delta\left(x_{i} \cdot \mu\right)^{1} \cong L\left(x_{i-1} \cdot \mu\right)$ and $\Delta\left(x_{i} \cdot \mu\right)^{2}=0$ for all $1 \leq i \leq n$ and $\mu \in C_{\text {fund }} \cap X$, so $\max \left\{j \mid \Delta\left(x_{i} \cdot \mu\right)^{j} \neq 0\right\}=1$. We have $x_{i}<x_{i} s_{n} \in W_{\text {aff }}^{+}$and Corollary 5.11 yields an upper bound on the length of the Jantzen filtration of $\Delta\left(x_{i} s_{n} \cdot \mu\right)$ :

$$
\max \left\{j \mid \Delta\left(x_{i} s_{n} \cdot \mu\right)^{j} \neq 0\right\} \leq 2 \cdot 1+1=3 .
$$


For the same Weyl module, the bound from Remark 5.13 yields

$$
\max \left\{j \mid \Delta\left(x_{i} s_{n} \cdot \mu\right)^{j} \neq 0\right\} \leq \ell\left(x_{i} s_{n}\right)=i+1 .
$$

We claim that $\max \left\{j \mid \Delta\left(x_{1} s_{n} \cdot \mu\right)^{j} \neq 0\right\}=1$ and $\max \left\{j \mid \Delta\left(x_{i} s_{n} \cdot \mu\right)^{j} \neq 0\right\}=2$ for $1<i \leq n$. Indeed, by the recursion formula and Lemma 6.1, we have

$$
\mathrm{JSF}_{x_{i} s_{n}}=N_{x_{i}}+\mathrm{JSF}_{x_{i}} \cdot s_{n}=N_{x_{i}}+\sum_{j=1}^{i-1}(-1)^{j+1} \cdot N_{x_{i-j} s_{n}}+(-1)^{i} \cdot N_{e}
$$

and as in Lemma 6.2, it follows that $\Delta\left(x_{1} s_{n} \cdot \mu\right)^{1} \cong L\left(x_{1} \cdot \mu\right)$ and $\Delta\left(x_{1} s_{n} \cdot \mu\right)^{2}=0$. Using Lemma 6.2 and induction on $i$, one sees that

$$
\sum_{j>0} \operatorname{ch} \Delta\left(x_{i} s_{n} \cdot \mu\right)^{j}=\operatorname{ch} L\left(x_{i} \cdot \mu\right)+\operatorname{ch} L\left(x_{i-1} s_{n} \cdot \mu\right)+2 \cdot L\left(x_{i-1} \cdot \mu\right)+\delta_{i, 2} \cdot \operatorname{ch} L(\mu)
$$

and

$\left[\Delta\left(x_{i} s_{n} \cdot \mu\right)\right]=\left[L\left(x_{i} s_{n} \cdot \mu\right)\right]+\left[L\left(x_{i} \cdot \mu\right)\right]+\left[L\left(x_{i-1} s_{n} \cdot \mu\right)\right]+\left[L\left(x_{i-1} s_{n} \cdot \mu\right)\right]+\delta_{i, 2} \cdot[L(\mu)]$

for $1<i \leq n$, where $\delta_{i, 2}=0$ if $i \neq 2$ and $\delta_{2,2}=1$. Note that (6.1) directly implies that the simple modules $L\left(x_{i} \cdot \mu\right)$ and $L\left(x_{i-1} s_{n} \cdot \mu\right)$ appear with multiplicity one in a composition series of the Weyl module $\Delta\left(x_{i} s_{n} \cdot \mu\right)$ (and so does $L(\mu)$ if $i=2$ ). The multiplicity of $L\left(x_{i-1} \cdot \mu\right)$ in $\Delta\left(x_{i} s_{n} \cdot \mu\right)$ equals the multiplicity of $L\left(x_{i-1} \cdot \mu\right)$ in $\Delta\left(x_{i} \cdot \mu\right)$ by Proposition 7.18 in [Jan03, and the latter multiplicity is also equal to one by Lemma 6.2. Combining (6.1) and (6.2), we see that $\Delta\left(x_{i} s_{n} \cdot \mu\right)^{2} \cong L\left(x_{i-1} \cdot \mu\right)$ and $\Delta\left(x_{i} s_{n} \cdot \mu\right)^{3}=0$, so $\max \left\{j \mid \Delta\left(x_{i} s_{n} \cdot \mu\right)^{j} \neq 0\right\}=2$ as claimed.

\section{ACKNOWLEDGMENTS}

The author would like to thank Donna Testerman and Thorge Jensen for numerous discussions and comments on the subject of this paper. Thanks are also due to Henning Haahr Andersen, Geordie Williamson and an anonymous referee for helpful comments on the manuscript.

\section{REFERENCES}

[AJS94] H. H. Andersen, J. C. Jantzen, and W. Soergel, Representations of quantum groups at a pth root of unity and of semisimple groups in characteristic $p$ : independence of $p$ (English, with English and French summaries), Astérisque 220 (1994), 321. MR.1272539

[AK08] Henning Haahr Andersen and Upendra Kulkarni, Sum formulas for reductive algebraic groups, Adv. Math. 217 (2008), no. 1, 419-447, DOI 10.1016/j.aim.2007.07.002. $\operatorname{MR} 2365202$

[And83] Henning Haahr Andersen, Filtrations of cohomology modules for Chevalley groups, Ann. Sci. École Norm. Sup. (4) 16 (1983), no. 4, 495-528 (1984). MR740588

[And87] Henning Haahr Andersen, Jantzen's filtrations of Weyl modules, Math. Z. 194 (1987), no. 1, 127-142. MR871225

[And97] Henning Haahr Andersen, Filtrations and tilting modules (English, with English and French summaries), Ann. Sci. École Norm. Sup. (4) 30 (1997), no. 3, 353-366, DOI 10.1016/S0012-9593(97)89924-7. MR1443491

[BB05] Anders Björner and Francesco Brenti, Combinatorics of Coxeter groups, Graduate Texts in Mathematics, vol. 231, Springer, New York, 2005. MR2133266

[Hum90] James E. Humphreys, Reflection groups and Coxeter groups, Cambridge Studies in Advanced Mathematics, vol. 29, Cambridge University Press, Cambridge, 1990, DOI 10.1017/CBO9780511623646. MR 1066460

[Jan77] Jens C. Jantzen, Darstellungen halbeinfacher Gruppen und kontravariante Formen, J. Reine Angew. Math. 290 (1977), 117-141, DOI 10.1515/crll.1977.290.117. MR 432775 
[Jan03] Jens Carsten Jantzen, Representations of algebraic groups, 2nd ed., Mathematical Surveys and Monographs, vol. 107, American Mathematical Society, Providence, RI, 2003. MR2015057

[KL93] D. Kazhdan and G. Lusztig, Tensor structures arising from affine Lie algebras. I, II, J. Amer. Math. Soc. 6 (1993), no. 4, 905-947, 949-1011, DOI 10.2307/2152745. MR.1186962

[KL94] D. Kazhdan and G. Lusztig, Tensor structures arising from affine Lie algebras. III, J. Amer. Math. Soc. 7 (1994), no. 2, 335-381, DOI 10.2307/2152762. MR1239506

[KT95] Masaki Kashiwara and Toshiyuki Tanisaki, Kazhdan-Lusztig conjecture for affine Lie algebras with negative level, Duke Math. J. 77 (1995), no. 1, 21-62, DOI 10.1215/S00127094-95-07702-3. MR1317626

[KT96] Masaki Kashiwara and Toshiyuki Tanisaki, Kazhdan-Lusztig conjecture for affine Lie algebras with negative level. II. Nonintegral case, Duke Math. J. 84 (1996), no. 3, 771813, DOI 10.1215/S0012-7094-96-08424-0. MR1408544

[Kul06] Upendra Kulkarni, A homological interpretation of Jantzen's sum formula, Transform. Groups 11 (2006), no. 3, 517-538, DOI 10.1007/s00031-005-1115-4. MR2264464

[Lus94] George Lusztig, Monodromic systems on affine flag manifolds, Proc. Roy. Soc. London Ser. A 445 (1994), no. 1923, 231-246, DOI 10.1098/rspa.1994.0058. MR1276910

[RW20] S. Riche and G. Williamson, Smith-Treumann theory and the linkage principle, preprint, URL: https://arxiv.org/abs/2003.08522, 2020.

[Sob20] Paul Sobaje, On character formulas for simple and tilting modules, Adv. Math. 369 (2020), 107172, 8, DOI 10.1016/j.aim.2020.107172. MR4092982

École Polytechnique Federale de Lausanne, 1015 Lausanne, Switzerland

Email address: jonathan.gruber@epfl.ch 